\title{
Radio continuum observations of new radio halos and relics from the NVSS and WENSS surveys
}

\section{Relic orientations, cluster X-ray luminosity, and redshift distributions}

\author{
R. J. van Weeren ${ }^{1}$, M. Brüggen ${ }^{3}$, H. J. A. Röttgering ${ }^{1}$, M. Hoeft ${ }^{2}$, S. E. Nuza ${ }^{4}$, and H. T. Intema ${ }^{5}$ \\ ${ }^{1}$ Leiden Observatory, Leiden University, PO Box 9513, 2300 RA Leiden, The Netherlands \\ e-mail: rvweeren@strw. leidenuniv.nl \\ 2 Thüringer Landessternwarte Tautenburg, Sternwarte 5, 07778, Tautenburg, Germany \\ 3 Jacobs University Bremen, PO Box 750561, 28725 Bremen, Germany \\ ${ }^{4}$ Astrophysikalisches Institut Potsdam, An der Sternwarte 16, 14482 Potsdam, Germany \\ 5 National Radio Astronomy Observatory, 520 Edgemont Road, Charlottesville, VA 22903-2475, USA
}

Received 27 April 2011 / Accepted 14 July 2011

\begin{abstract}
Context. Radio halos and relics are diffuse radio sources found in galaxy clusters showing significant substructure at X-ray wavelengths. These sources provide important information about non-thermal processes taking place in the intracluster medium (ICM). Until now only a few dozen relics and halos are known, while models predict that a much larger number of these sources exist. In this paper we present the results of an extensive observing campaign to search for new diffuse radio sources in galaxy clusters.

Aims. The aim of the observations is to create a large sample of diffuse radio sources in galaxy clusters that help to understand the formation of radio relics and halos and can be used to probe the physical conditions of the ICM.

Methods. We carried out radio continuum observations with the Westerbork Synthese Radio Telescope (WSRT), Giant Metrewave Radio Telescope (GMRT) and Very Large Array (VLA) of clusters with diffuse radio emission visible in NVSS and WENSS survey images. Optical images were taken with the William Herschel and Isaac Newton Telescope (WHT, INT).

Results. We discovered 6 new radio relics, including a probable double relic system, and 2 radio halos. In addition, we confirm the presence of diffuse radio emission in four galaxy clusters. By constructing a sample of 35 radio relics we find that relics are mostly found along the major axis of the X-ray emission from the ICM, while their orientation is perpendicular to this axis. We also compared the X-ray luminosity and redshift distributions of clusters with relics to an X-ray selected sample from the NORAS and REFLEX surveys. We find tentative evidence for an increase of the cluster's relic fraction with X-ray luminosity and redshift. The major and minor axis ratio distribution of the ICM for clusters with relics is broader than that of the NORAS-REFLEX sample.

Conclusions. The location and orientation of radio relics with respect to the ICM elongation is consistent with the scenario that relics trace merger shock waves.
\end{abstract}

Key words. galaxies: clusters: general - galaxies: clusters: intracluster medium - radio continuum: galaxies -

large-scale structure of Universe

\section{Introduction}

Radio halos and relics are found in massive merging galaxy clusters. These radio sources indicate the presence of magnetic fields and in-situ particle acceleration within the ICM (e.g., Jaffe 1977; Govoni \& Feretti 2004). Galaxy clusters form through mergers with other clusters and galaxy groups as well as through continuous accretion of gas from the intergalactic medium. Since giant radio halos and relics are found in merging clusters (e.g., Buote 2001; Govoni et al. 2004; Barrena et al. 2007; Brunetti et al. 2009; Cassano et al. 2010b), it has been proposed that a small fraction of the energy released during a cluster merger event is channeled into the (re)acceleration of particles.

One scenario for the origin of radio relics is that they trace merger shock waves within the ICM in which particles are accelerated by the diffusive shock acceleration (DSA) mechanism (Krymskii 1977; Axford et al. 1977; Bell 1978a,b; Blandford \& Ostriker 1978; Drury 1983; Blandford \& Eichler 1987; Jones \& Ellison 1991; Malkov \& O'C Drury 2001). In the presence of a magnetic field these particles emit synchrotron radiation at radio wavelengths (e.g., Enßlin et al. 1998; Miniati et al. 2000). The efficiency with which collisionless shocks can accelerate particles is unknown and may not be enough to produce the observed radio brightness of relics. A closely linked scenario is that of shock re-acceleration of pre-accelerated electrons in the ICM, which is a more efficient mechanism for weak shocks (e.g., Markevitch et al. 2005; Giacintucci et al. 2008; Kang \& Ryu 2011).

An alternative scenario has been proposed by Keshet (2010) based on a secondary cosmic ray electron model, where the time evolution of magnetic fields and the cosmic ray distribution are taken into account to explain both halos and giant relics. Detailed spectral maps, at multiple frequencies, and measurements of the magnetic field via the polarization properties can test this model. For relics, strong magnetic fields $\left(\boldsymbol{B}>\boldsymbol{B}_{\mathrm{cmb}}=3.25 \times(1+z)^{2}\right)$ are predicted and the spectral index ${ }^{1}$ at the outer edges of relics should be flat, with $\alpha \simeq-1$.

\footnotetext{
${ }^{1} F_{v} \propto v^{\alpha}$, where $\alpha$ is the spectral index.
} 
A few clusters host so-called double relics systems (van Weeren et al. 2011a; Brown et al. 2011; van Weeren et al. 2010; Bonafede et al. 2009b; van Weeren et al. 2009a; Venturi et al. 2007; Feretti et al. 2005; Bagchi et al. 2006; Röttgering et al. 1997), with both relics located symmetrically about to the cluster center and along the merger axis, as indicated by X-ray observations of the thermal ICM. Another class of diffuse filamentary radio sources in clusters are radio "phoenices". These sources have extremely steep radio spectra $(\alpha \lesssim-1.5)$ probably due to synchrotron and inverse Compton (IC) losses. The idea is that fossil radio plasma from a previous episode of AGN activity is compressed by a merger shock wave producing a radio phoenix (Enßlin \& Gopal-Krishna 2001; Enßlin \& Brüggen 2002). Related to phoenices are "AGN relics", which consist of radio plasma from extinct AGN.

Unlike relics, radio halos are found in the center of merging galaxy clusters and follow the X-ray emission from the ICM. Radio halos have been explained by turbulence injected by recent merger events. The injected turbulence is thought to be capable of re-accelerating relativistic particles (e.g., Brunetti et al. 2001; Petrosian 2001; Cassano \& Brunetti 2005). Alternatively, the energetic electrons are secondary products which originate from hadronic collisions between relativistic protons and thermal ions (e.g., Dennison 1980; Blasi \& Colafrancesco 1999; Dolag \& Enßlin 2000; Keshet \& Loeb 2010). Recent observations put tension on the secondary models (e.g, Donnert et al. 2010a,b; Jeltema \& Profumo 2011; Brown \& Rudnick 2011; Bonafede et al. 2011). The existence of ultra-steep spectrum radio halos is also not in agreement with these secondary models (Brunetti et al. 2008), but they can be explained by the turbulent re-acceleration model. However, currently only a few of these ultra-steep spectrum radio halos are known so more observations, such as presented in this paper, are needed to increase this number and provide better measurements of the radio spectra.

In the last decade a number of successful searches have been carried out to find new diffuse radio sources in galaxy clusters (e.g., Giovannini et al. 1999; Giovannini \& Feretti 2000; Govoni et al. 2001; Kempner \& Sarazin 2001; Bacchi et al. 2003; Venturi et al. 2007, 2008; Giovannini et al. 2009; van Weeren et al. 2009b; Rudnick \& Lemmerman 2009). However, our understanding of the formation of these sources is still limited. Models for the formation of relics and halos can be tested through statistical studies of correlations between the non-thermal radio emission and global properties of the clusters, such as mass, temperature, and dynamical state (Liang et al. 2000; Feretti et al. 2006; Cassano et al. 2006, 2007, 2008, 2010a).

We recently discovered two large radio relics in the NVSS and WENSS surveys (van Weeren et al. 2010, 2011a). Interestingly, these relics remained unnoticed for about 15 years. This suggests that more diffuse radio sources could be discovered by inspection of the NVSS (Condon et al. 1998) and WENSS (Rengelink et al. 1997) survey images. We therefore carried out a visual inspection of the NVSS and WENSS images around known clusters detected by ROSAT (Voges et al. 1999, 2000; Ebeling et al. 1998; Böhringer et al. 2000; Kocevski et al. 2007; Ebeling et al. 2002; Böhringer et al. 2004).

Candidate clusters hosting diffuse radio emission were observed with the WSRT, GMRT and/or VLA. Clusters with existing published observations were not re-observed. In this paper we present the radio images and global properties of the clusters. In addition, we investigate the position and orientation of radio relics with respect the ICM, and compare the X-ray luminosity and redshift distributions of clusters with relics to an $\mathrm{X}$-ray selected sample. In a follow-up paper we will present the polarization and detailed spectral properties of the radio emission in these clusters. The layout of this paper is as follows. In Sect. 2 we give an overview of the observations and the data reduction. In Sect. 3 we present the radio images and give an short overview of the cluster's properties. We end with discussions and conclusions in Sects. 4 and 5.

Throughout this paper we assume a $\Lambda \mathrm{CDM}$ cosmology with $H_{0}=71 \mathrm{~km} \mathrm{~s}^{-1} \mathrm{Mpc}^{-1}, \Omega_{\mathrm{m}}=0.27$, and $\Omega_{\Lambda}=0.73$. All images are in the $\mathrm{J} 2000$ coordinate system.

\section{Observations and data reduction}

\subsection{Radio observations}

Most of candidate clusters with diffuse radio emission were observed with the WSRT. GMRT or VLA observations were taken of a few clusters which were missed by the WSRT observations. A summary of the observations is given in Table 1. The WSRT observations were taken in frequency switching mode, alternating every 5 min between the 21 and $18 \mathrm{~cm}$ bands. In this paper we will only use the $21 \mathrm{~cm}$ data, except for the clusters Abell 697 and Abell 2061. The other data will be presented in a future paper which will focus on the polarization properties and rotation measure synthesis. The total integration time for the WSRT observations was $6 \mathrm{~h}$ per cluster, except for Abell 3365 (see Table 1). VLA observations of Abell 523 were taken in D-array. We also included archival observations from project AB1180 ( $L$ band D and C-array). Abell 3365 L-band VLA observations were taken in DnC and CnB array (project AR690).

GMRT $325 \mathrm{MHz}$ observations (with the hardware correlator) were taken of Abell 1612 on May 13, 2009, recording both RR and LL polarizations with $32 \mathrm{MHz}$ bandwidth. We observed Abell 1612 and CIZA J0649.3+1801 at 610/241 MHz with the GMRT. The 610/241 MHz observations were taken in dualfrequency mode, recording RR polarization at $610 \mathrm{MHz}$ and $\mathrm{LL}$ polarization at $241 \mathrm{MHz}$. Total recorded bandwidth was $32 \mathrm{MHz}$ at $610 \mathrm{MHz}$ and $6 \mathrm{MHz}$ at $241 \mathrm{MHz}$. The GMRT software backend (GSB; Roy et al. 2010) was used.

For the data reduction we used the NRAO Astronomical Image Processing System (AIPS) package. Standard gain calibration and RFI removal were performed. Bandpass calibration was carried out for observations done in spectral line mode. For the $241 \mathrm{MHz}$ observations RFI was fitted and subtracted from the data using the technique described by Athreya (2009) which was implemented in Obit (Cotton 2008). The fluxes of the primary calibrators were set according to the Perley \& Taylor (1999) extension to the Baars et al. (1977) scale. Several rounds of phase self-calibration were performed before doing two final rounds of amplitude and phase self-calibration.

For GMRT data in the imaging step we used the polyhedron method (Perley 1989; Cornwell \& Perley 1992) to minimize the effects of non-coplanar baselines. Images were made using "Briggs" weighting (with robust set to 0.5, Briggs 1995) and cleaned down to 2 times the rms noise level $\left(2 \sigma_{\text {rms }}\right)$ using clean boxes. Finally, we corrected the image for the primary beam attenuation. For more details on the data reduction the reader is referred to van Weeren et al. (2011b).

\subsection{Optical WHT \& INT images}

For the clusters Abell 1612, Abell 523, Abell 3365, Abell 2034, CIZA J0649.3+1801 and RX J0107.8+5408 we made use of optical V, R and I band images taken with the WHT (PFIP camera) and INT (WFC camera) telescopes between 15-19 April (WHT) 
Table 1. Observations.

\begin{tabular}{|c|c|c|c|c|c|c|}
\hline Cluster & Observation date & $\begin{array}{l}\text { Frequency } \\
\mathrm{MHz}\end{array}$ & $\begin{array}{c}\text { Bandwidth } \\
\text { MHz }\end{array}$ & $\begin{array}{c}\text { Integration time } \\
\mathrm{hr}\end{array}$ & $\begin{array}{l}\text { Map resolution } \\
\text { arcsec }\end{array}$ & $\begin{array}{l}\text { rms noise } \\
\mu \mathrm{Jy} \mathrm{beam}^{-1}\end{array}$ \\
\hline Abell 1612 & GMRT, 13 May, 2009 & 325 & 32 & 4.0 & $11.6^{\prime \prime} \times 9.5^{\prime \prime}$ & 236 \\
\hline & GMRT, 22 Nov, 2009 & 610,241 & 32,6 & $2.5,2.5$ & $7.7^{\prime \prime} \times 4.7^{\prime \prime}, 21^{\prime \prime} \times 12^{\prime \prime}$ & 64,777 \\
\hline Abell 523 & $\begin{array}{c}\text { VLA Aug, 25, 2005; } \\
\text { Nov 28, 2005; Dec 28, } 2009\end{array}$ & 1425 & 37.5 & $1.1^{a 1}, 3.7^{a 2}$ & $21^{\prime \prime} \times 20^{\prime \prime d}$ & 40 \\
\hline Abell $697^{e}$ & WSRT, 24 Aug, 2009 & 1382,1714 & 160,160 & $6.0,6.0$ & $34^{\prime \prime} \times 21^{\prime \prime b}$ & 24,32 \\
\hline Abell $3365^{e}$ & WSRT, 22 Feb, 2009 & 1382 & 160 & 12.0 & $108^{\prime \prime} \times 13^{\prime \prime}$ & 29 \\
\hline Abell 3365 & $\begin{array}{l}\text { VLA, } 30 \text { Sep, } 2009 \\
30 \text { May, } 2009\end{array}$ & 1425 & $2 \times 50$ & $0.7^{g 1}, 2.5^{g 2}$ & $43^{\prime \prime} \times 35^{\prime \prime}, 13.5^{\prime \prime} \times 9.2^{\prime \prime}$ & 84,27 \\
\hline Abell $746^{e}$ & WSRT, 19 Sep, 2009 & 1382 & 160 & 6.0 & $23^{\prime \prime} \times 18^{\prime \prime}$ & 28 \\
\hline Abell $2034^{f}$ & WSRT, 26 Jul, 2009 & 1382 & 160 & 6.0 & $30^{\prime \prime} \times 16^{\prime \prime}$ & 24 \\
\hline Abell $2061^{f}$ & WSRT, 23 Jul, 2009 & 1382,1714 & 160,160 & $6.0,6.0$ & $32^{\prime \prime} \times 16^{\prime \prime b}$ & 22,26 \\
\hline RXC J1053.7+5452e & WSRT, 14 Mar, 2009 & 1382 & 160 & 6.0 & $24^{\prime \prime} \times 18^{\prime \prime}$ & 30 \\
\hline CIZA J0649.3+1801 & GMRT, 22 Nov, 2009 & 610,241 & 32,6 & $3.0,3.0$ & $25^{\prime \prime} \times 25^{\prime \prime c}, 17^{\prime \prime} \times 14^{\prime \prime}$ & 515,1800 \\
\hline RX J0107.8+5408 & WSRT, 29 Aug, 2009 & 1382 & 160 & 6.0 & $21^{\prime \prime} \times 17^{\prime \prime}$ & 29 \\
\hline
\end{tabular}

Notes. ${ }^{a 1} \mathrm{D}$ array; ${ }^{a 2} \mathrm{C}$ array; ${ }^{b}$ the WSRT $1714 \mathrm{MHz}$ image was convolved to the same resolution as the $1382 \mathrm{MHz}$ image; ${ }^{c}$ convolved to a beam of $25^{\prime \prime} \times 25^{\prime \prime} ;{ }^{d} \mathrm{D}$ and C array data were combined; ${ }^{e}$ the minimum baseline length is $36 \mathrm{~m} ;{ }^{f}$ the minimum baseline length is $54 \mathrm{~m} ;{ }^{g 1} \mathrm{DnC}$ array; ${ }^{2} \mathrm{CnB}$ array.

and 1-8 October (INT), 2009 (for more details see van Weeren et al. 2011b).

\section{Results}

In this section we present the radio continuum images of the clusters. We briefly discuss the results of the radio observations for each cluster, a summary is given in Table 2 . To compute the integrated fluxes for the diffuse radio sources we subtracted the flux contribution from the discrete sources. We alphabetically labeled these discrete sources for each cluster where applicable. The fluxes of the discrete sources are reported in Table 3 and they are measured from images made with uniform weighting. We included the uncertainties in the subtraction of the discrete sources in the uncertainty for the integrated flux measurements of the diffuse cluster emission in Table 2. We assume that the uncertainties for the discrete sources are uncorrelated. More details are given in the subsections of the individual clusters.

We also display overlays onto ROSAT images and galaxy iso-density contours (mostly derived from SDSS DR7 photometric redshifts, Abazajian et al. 2009). The iso-densities were computed by counting the number of galaxies per unit surface area within a certain photometric redshift range (for SDSS) or $V-R$ and $R-I$ color range (for INT images). For the INT images we created a catalog of objects using Sextractor (Bertin $\&$ Arnouts 1996). We then removed all point-like objects (i.e., stars) from the catalogs. To exclude galaxies not belonging to the cluster we selected only galaxies with $R-I$ and $V-R$ colors within $0.15 \mathrm{mag}$ from the average color of a massive elliptical $\mathrm{cD}$ galaxy in the cluster. The range of 0.15 in the colors was taken to maximize the contrast of the cluster with respect to the fore and background galaxies in the field, but not being too restrictive so that a sufficient number of candidate cluster members was selected.

\subsection{Abell 1612}

Abell 1612 is a little studied cluster at $z=0.179$ (Popesso et al. 2004) with a moderate X-ray luminosity of $L_{\mathrm{X}, 0.1-2.4 \mathrm{keV}}=2.41 \times 10^{44} \mathrm{erg} \mathrm{s}^{-1}$ (Böhringer et al. 2004). In the NVSS survey we found an elongated radio source located about $5^{\prime}$ to the south of the cluster center. The source was completely resolved out in the $1.4 \mathrm{GHz} 5$ " FIRST survey (Becker et al. 1995) indicating diffuse emission on scales of about $4^{\prime}$. In our GMRT $325 \mathrm{MHz}$ image (Fig. 1, left panel) the source has a total extent of $4.5^{\prime}$, which corresponds to a physical size of $780 \mathrm{kpc}$. We could not identify an optical counterpart for the source (Fig. 3, left panel). We therefore classify the source as a radio relic. In the $610 \mathrm{MHz}$ image (Fig. 2, left panel) the source is connected to a tailed radio galaxy to the north. Combining flux measurements at $1.4 \mathrm{GHz}$ (NVSS) and GMRT fluxes at 241, 325 and $610 \mathrm{MHz}$ gives $\alpha \approx-1.4$. However, we note that the individual flux measurements give a large scatter (Fig. 3, right panel). We checked the absolute flux calibration between the different frequencies by measuring the integrated fluxes of several compact sources. This did not reveal any problems. Some of the short baselines in the 325 and $241 \mathrm{MHz}$ observations were affected by RFI which could have affected the flux measurement since the source is quite extended. In addition, the declination is close to $0^{\circ}$ which gives a non-optimal uv-coverage.

Probably the relic traces a shock in which particles are accelerated or re-accelerated by a merger-related shock wave. The large extent makes it unlikely that the source traces compressed fossil radio plasma (Clarke \& Ensslin 2006). The connection with the tailed radio source to the north favors a re-acceleration scenario. In this case, the seed relativistic electrons could be supplied by the tailed-radio source. The elongated galaxy isodensity contours and ICM, see Fig. 1 (right panel), also hint at a merger event along a northwest-southeast axis.

\subsection{Abell 746}

Abel 746 is a Bautz-Morgan (B/M) class III type cluster located at $z_{\text {phot }}=0.232 \pm 0.01$ (Koester et al. 2007). Based on the ROSAT flux from Böhringer et al. (2000) we calculate $L_{\mathrm{X}, 0.1-2.4 \mathrm{keV}} \sim 3.68 \times 10^{44} \mathrm{erg} \mathrm{s}^{-1}$. We discovered an elongated radio source located northwest of the cluster center in the NVSS survey. The diffuse source is resolved out in the FIRST image, except for an unresolved source located at the southern tip of the diffuse source. In our WSRT image the elongated source is easily detected (Fig. 5, left panel). The source has a largest angular extent of $5^{\prime}$, which corresponds to a physical size of $1.1 \mathrm{Mpc}$ at the distance of Abell 746. The source has a width of $345 \mathrm{kpc}$ and is located at a distance of $1.7 \mathrm{Mpc}$ from the cluster center. 
Table 2. Cluster properties.

\begin{tabular}{|c|c|c|c|c|c|c|}
\hline Relic & $z$ & $\begin{array}{c}S_{1382 \mathrm{MHz}} \\
\mathrm{mJy}\end{array}$ & $\begin{array}{c}P_{1.4 \mathrm{GHz}} \\
10^{24} \mathrm{~W} \mathrm{~Hz}^{-1}\end{array}$ & $\begin{array}{c}L_{\mathrm{X}, 0.1-2.4 \mathrm{keV}^{d}} \\
10^{44} \mathrm{erg} \mathrm{s}^{-1}\end{array}$ & $\begin{array}{c}\mathrm{LLS}^{a} \\
\mathrm{kpc}\end{array}$ & Classification $^{b}$ \\
\hline Abell 1612 & 0.179 & $62.8 \pm 2.6^{e}$ & 7.9 & 2.41 & 780 & $\mathrm{R}$ \\
\hline Abell 746 (center, periphery) & 0.232 & $18 \pm 4,24.5 \pm 2.0$ & $3.8,6.8$ & 3.68 & 850,1100 & $\mathrm{H}, \mathrm{R}$ \\
\hline Abell 523 & 0.10 & $61 \pm 7^{f}$ & 1.7 & 0.89 & 1350 & $\mathrm{H}$ or $\mathrm{R}$ \\
\hline Abell 697 & 0.282 & $5.2 \pm 0.5,4.0 \pm 0.5^{g}$ & 2.2 & 19.42 & 750 & $\mathrm{H}$ \\
\hline Abell 2061 & 0.0784 & $27.6 \pm 1.0,21.2 \pm 2.1^{g}$ & 0.45 & 3.95 & 675 & $\mathrm{R}$ \\
\hline Abell 3365 & 0.0926 & $\begin{array}{c}42.4 \pm 3.0^{j}, 5.4 \pm 0.5^{k} \\
42.6 \pm 2.6^{f, j}, 5.3 \pm 0.5^{f, k}\end{array}$ & $1.1^{j}, 0.14^{k}$ & 0.859 & $560^{j}, 235^{k}$ & DR \\
\hline CIZA J0649.3+1801 & 0.064 & $321 \pm 46^{i}$ & $1.2^{h}$ & 2.38 & 800 & $\mathrm{R}$ \\
\hline RX J0107.8+5408 & 0.1066 & $55 \pm 5$ & 1.8 & 5.42 & 1100 & $\mathrm{H}$ \\
\hline Abell 2034 (center, periphery) & 0.113 & $7.3 \pm 2.0,24 \pm 2$ & $0.28,0.89$ & 3.56 & 600,220 & $\mathrm{H}$ or $\mathrm{R}, \mathrm{R}$ \\
\hline RXC J1053.7+5452 & 0.0704 & $15 \pm 2$ & 0.2 & 0.44 & 600 & $\mathrm{R}$ \\
\hline
\end{tabular}

Notes. ${ }^{a}$ Largest linear size $;{ }^{b} \mathrm{R}=$ radio relic, $\mathrm{H}=$ radio halo, $\mathrm{DR}=$ double radio relic; ${ }^{c}$ also known as CIZA J0107.7+5408 or ZwCl 0104.9+5350; ${ }^{d}$ Abell 3365 and Abell 1612: Böhringer et al. (2004), CIZA J0649.3+1801 and RX J0107.8+5408: Ebeling et al. (2002), RXC J1053.7+5452: Popesso et al. (2004), Abell 697: Popesso et al. (2004), Abell 2034 and Abell 2061: Ebeling et al. (1998), Abell 523: Böhringer et al. (2000), Abell 746: this work; ${ }^{e}$ NVSS flux; ${ }^{f}$ VLA flux at $1425 \mathrm{MHz} ;{ }^{g}$ WSRT flux at $1714 \mathrm{MHz} ;{ }^{h}$ using a typical relic spectral index of $-1.3 ;{ }^{i}$ GMRT flux at $610 \mathrm{MHz} ;{ }^{j}$ eastern relic; ${ }^{k}$ western relic.

Table 3. Fluxes of compact sources embedded in the diffuse emission.

\begin{tabular}{|c|c|c|c|c|c|c|c|c|c|c|}
\hline & $\bar{A}$ & $\overline{\mathrm{B}}$ & $\overline{\mathrm{C}}$ & $\overline{\mathrm{D}}$ & $\bar{E}$ & $\bar{F}$ & $\overline{\mathrm{H}}$ & $\mathrm{I}$ & $\mathrm{J}$ & Figure \\
\hline A746 & $1.52 \pm 0.09$ & $0.36 \pm 0.06$ & $0.24 \pm 0.06$ & $0.37 \pm 0.06$ & $0.45 \pm 0.07$ & $0.93 \pm 0.06$ & $0.51 \pm 0.07$ & $0.33 \pm 0.06$ & $0.34 \pm 0.06$ & 5 \\
\hline A523 & $10.9 \pm 0.2$ & $5.7 \pm 0.1$ & $2.9 \pm 0.1$ & & & & & & & 6 \\
\hline $\mathrm{A} 697^{a}$ & $0.50 \pm 0.07$ & $0.29 \pm 0.06$ & $0.22 \pm 0.06$ & $0.25 \pm 0.08$ & $0.16 \pm 0.06$ & $0.19 \pm 0.05$ & & & & 9 \\
\hline $\mathrm{A} 697^{b}$ & $0.38 \pm 0.08$ & $0.24 \pm 0.07$ & $0.17 \pm 0.05$ & $0.12 \pm 0.05^{d}$ & $0.15 \pm 0.06^{d}$ & $0.07 \pm 0.05^{d}$ & & & & 9 \\
\hline $\mathrm{A} 2061^{a}$ & $0.53 \pm 0.08$ & $0.41 \pm 0.07$ & $0.36 \pm 0.07$ & & & & & & & 13 \\
\hline $\mathrm{A} 2061^{b}$ & $0.31 \pm 0.08^{d}$ & $0.33 \pm 0.08$ & $0.20 \pm 0.08^{d}$ & & & & & & & 13 \\
\hline $\mathrm{A} 3365^{c}$ & $1.5 \pm 0.1$ & $0.53 \pm 0.06$ & $0.62 \pm 0.06$ & & & & & & & 15 \\
\hline CIZA J0649.3+1801 & $7.7 \pm 0.8$ & $1.2 \pm 0.1$ & $2.5 \pm 0.3$ & & & & & & & 17 \\
\hline RX J0107.8+5408 & $2.6 \pm 0.2$ & $1.1 \pm 0.1$ & $1.0 \pm 0.1$ & $0.7 \pm 0.1$ & & & & & & 18 \\
\hline A2034 & $0.93 \pm 0.06$ & $0.59 \pm 0.06$ & $0.34 \pm 0.05$ & & & & & & & 19 \\
\hline RXC J1053.7+5452 & $1.2 \pm 0.1$ & $1.4 \pm 0.1$ & & & & & & & & 21 \\
\hline
\end{tabular}

Notes. Reported fluxes are in mJy; ${ }^{a} 1382 \mathrm{MHz} ;{ }^{b} 1714 \mathrm{MHz} ;{ }^{c}$ from VLA CnB array image; ${ }^{d}$ by measuring the flux at the $1382 \mathrm{MHz}$ source position.
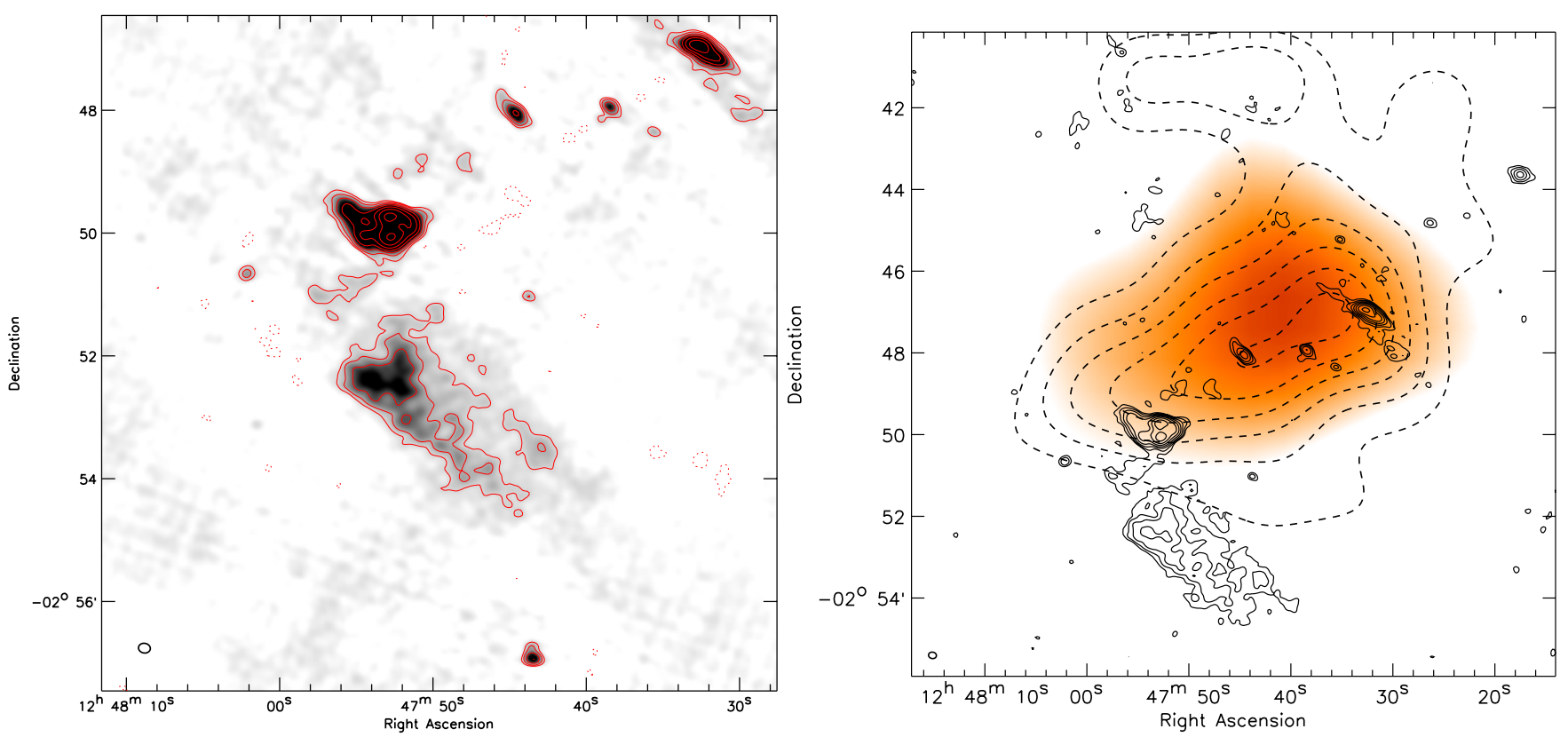

Fig. 1. Left: GMRT $325 \mathrm{MHz}$ image of Abell 1612. Radio contours are drawn at levels of $[1,2,4, \ldots] \times 4 \sigma_{\text {rms }}$. Dashed contours are drawn at $-3 \sigma_{\text {rms }}$. Right: A1612 X-ray emission from ROSAT, tracing the thermal ICM, is shown by the color image. The original image from the ROSAT All Sky Survey was convolved with a 270" FWHM Gaussian. Solid contours are from the GMRT $325 \mathrm{MHz}$ image and drawn at levels of [1,2, 4,...] $\times$ $3 \sigma_{\text {rms. }}$. Dashed contours show the galaxy iso-density distribution derived from the SDSS survey. Contours are drawn at $[1.0,1.4,1.8, \ldots] \times 1.1$ galaxies $\operatorname{arcmin}^{-2}$ selecting only galaxies with $0.16<z_{\text {phot }}<0.20$. 
R. J. van Weeren et al.: Radio continuum observations of new radio halos and relics from the NVSS and WENSS surveys
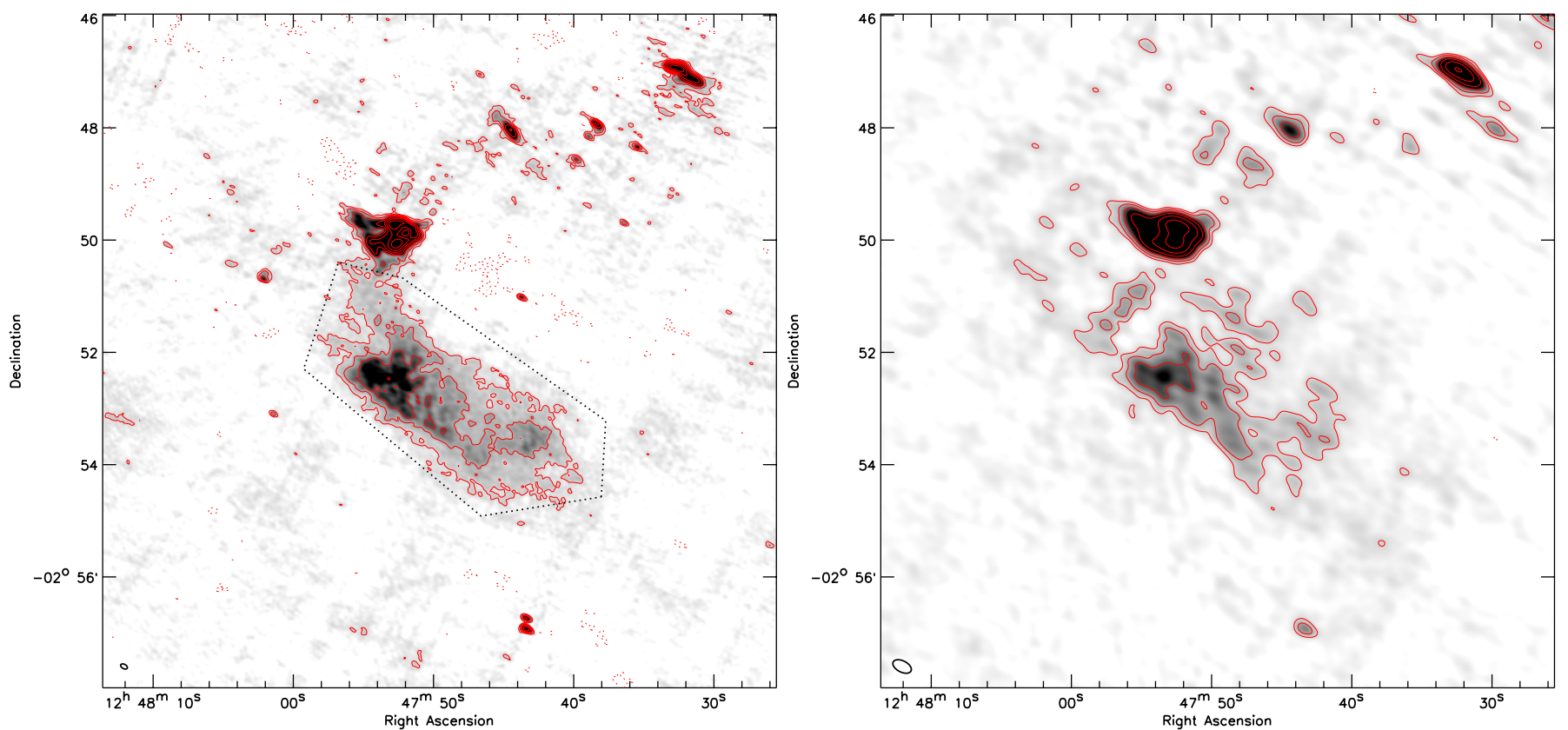

Fig. 2. GMRT 610 (left) and $241 \mathrm{MHz}$ (right) image of A1612. Contour levels are drawn as in Fig. 1. Black dotted lines in the $610 \mathrm{MHz}$ image indicate the integration area for the flux measurements.
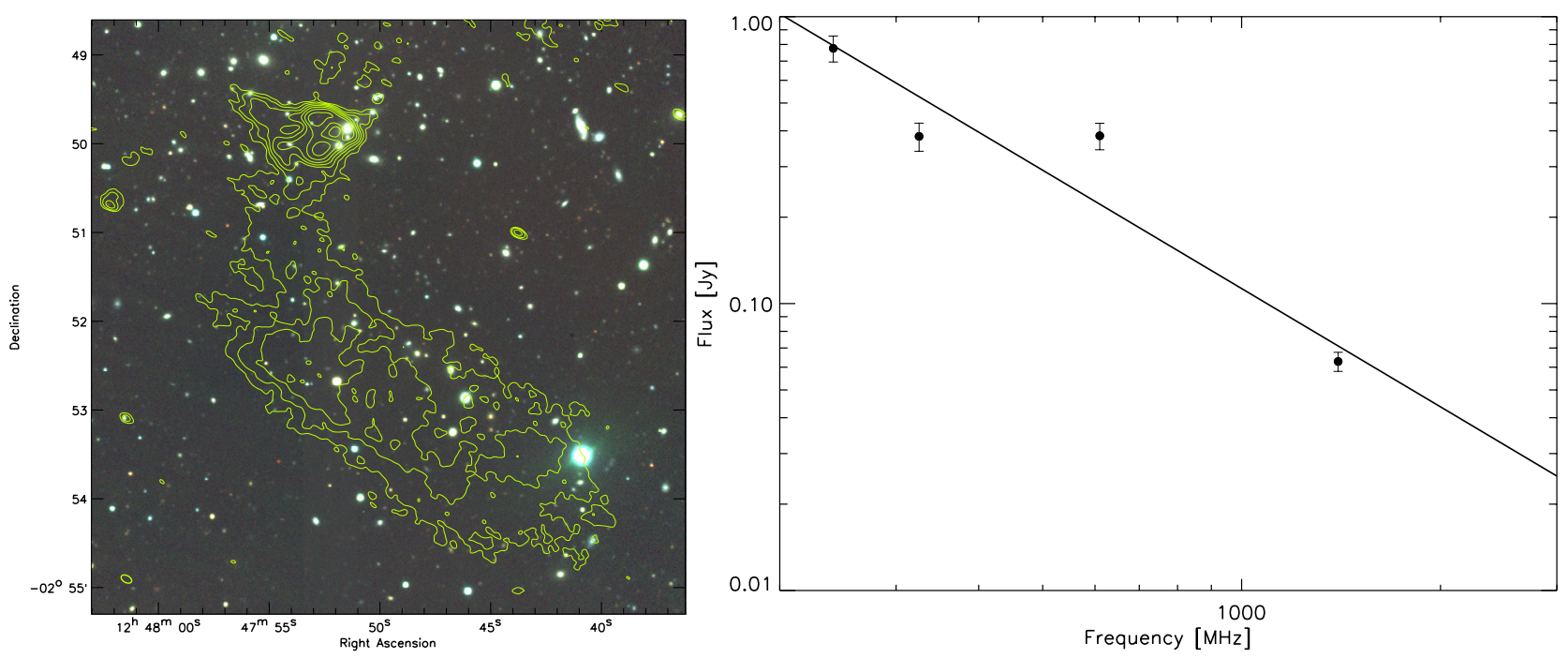

Fig. 3. Left: WHT $V, R, I$ color image of Abell 1612. Overlaid are the radio contours from Fig. 2 (left panel). Right: A1612 radio relic spectrum. Flux measurements at 241, 325, and $610 \mathrm{MHz}$ are from the GMRT observations. The $1.4 \mathrm{GHz}$ flux is from the NVSS survey image.

We do not find it very likely that the point source at the southern end of the elongated source is the hotspot of a radio galaxy because the second hotspot is missing. In addition, we do not detect any radio core. The compact source at the southern end of the diffuse source does not have an optical counterpart in SDSS images. The SDSS images are contaminated by the bright star f Uma $\left(V_{\mathrm{mag}}=4.5\right)$. We only conclude that a possible optical counterpart should be located farther away than A746. This limit on the redshift does not provide any useful constraints to rule it out as a giant radio galaxy on the basis of its physical size or radio luminosity. The morphology of the source is more typical for a radio relic. The size of the sources an its location would also agree with this interpretation. As an additional check, we analyzed the polarization data from the WSRT observation. Radio relics are often polarized at a level of $\sim 20 \%$ or more (e.g., Clarke \& Ensslin 2006; Bonafede et al. 2009b; van Weeren et al. 2010). Indeed, we find that the elongated source is polarized up to the $\sim 50 \%$ level (Fig. 4), which provides additional support for the classification as radio relic.

Galaxy iso-density contours show the cluster to be somewhat elongated along a northwest-southeast axis, see Fig. 5 (right panel). The ROSAT image reveals little structure. However, the number of photons detected from the cluster is low.

The WSRT image reveals additional diffuse emission in the center of the cluster, albeit at a low SNR. The emission has an extent of at least $850 \mathrm{kpc}$ which is typical for a giant radio halo. The flux of this radio halo is difficult to estimate due to the low SNR, but subtracting the contribution from the compact sources we 


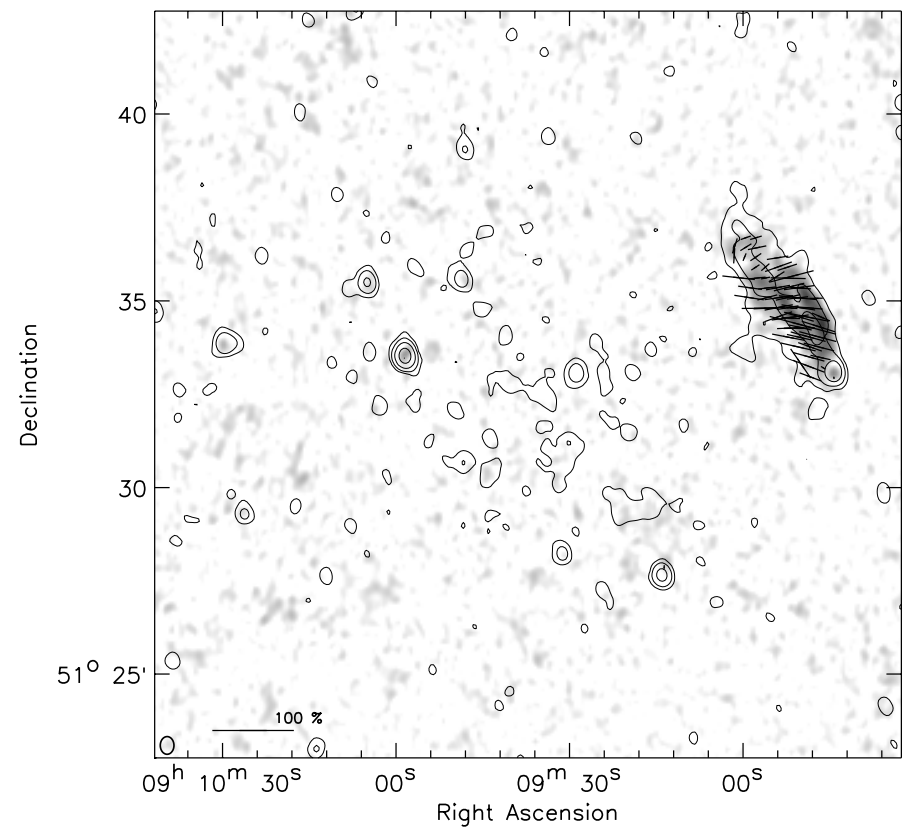

Fig. 4. WSRT $1382 \mathrm{MHz}$ polarization map of A746. Total polarized intensity is shown as a grayscale image. Vectors depict the polarization $E$-vectors, their length represents the polarization fraction. The length of the $E$-vectors are corrected for Ricean bias (Wardle \& Kronberg 1974). A reference vector for a polarization fraction of $100 \%$ is shown in the bottom left corner. No vectors were drawn for pixels with a $S N R<4$ in the total polarized intensity image. Contour levels are drawn at $[1,4,16,64, \ldots] \times 4 \sigma_{\text {rms }}$ and are from the Stokes $I$ image.

find a flux of about $18 \pm 4 \mathrm{mJy}$ for the radio halo. To better image the diffuse emission we subtracted the clean components from the compact sources using an image made with uniform weighting. We then convolved the image (made with natural weighting) to a resolution of $1^{\prime}$. The contours from this image are overlaid in Fig. 5 (right panel). The halo is now better detected and the radio emission roughly follows the $\mathrm{X}$-ray emission. The radio power of $3.8 \times 10^{24} \mathrm{~W} \mathrm{~Hz}{ }^{-1}$ is above the $L_{\mathrm{X}}-P_{1.4 \mathrm{GHz}}$ correlation for giant radio halos (e.g., Liang et al. 2000; Cassano et al. 2006). The fitted relation from Cassano et al. (2006) gives a power of $0.64 \times 10^{24} \mathrm{~W} \mathrm{~Hz}^{-1}$. We note that both the integrated radio flux and $L_{\mathrm{X}}$ (from ROSAT) are uncertain, and that the intrinsic scatter in the $L_{\mathrm{X}}-P_{1.4 \mathrm{GHz}}$ relation is quite large (Brunetti et al. 2009). The measured radio power is therefore still marginally consistent with being on the $L_{\mathrm{X}}-P_{1.4 \mathrm{GHz}}$ correlation.

\subsection{Abell 523}

Abel 523 is a little studied galaxy cluster located at $z=0.10$ (Struble \& Rood 1999), with $L_{\mathrm{X}}, 0.1-2.4 \mathrm{keV}=0.89 \times 10^{44} \mathrm{erg} \mathrm{s}^{-1}$ (Böhringer et al. 2000). Galaxy iso-densities derived from INT images show a north-south elongated cluster consisting of two galaxy clumps. The VLA image (Fig. 6, left panel) reveals a large, irregular and diffuse radio source in the cluster as well as a number of compact sources related to AGN activity. Our radio image and galaxy distribution agree with the recent results from Giovannini et al. (2011) which showed the presence of diffuse radio emission in this cluster.

The brightest radio source is a tailed radio galaxy projected relatively close to the cluster center. The color of the optical counterpart is similar to other galaxies in the cluster making it likely that the radio source is associated with cluster. The optical INT image is shown in Fig. 7. We also detect some radio emission from the largest $\mathrm{cD}$ galaxy in the cluster located north of the tailed radio source. In the southern part of the cluster there is a brighter compact source associated with another large elliptical galaxy.

The diffuse source has a patchy morphology, with the brightest part of the diffuse source located to the northwest of the tailed radio source. To the west the diffuse source extends into two filamentary structures. The total flux of the diffuse radio source, minus the point sources and head-tail galaxy, is $61 \pm 7 \mathrm{mJy}$. The diffuse source has a largest extent of $1.35 \mathrm{Mpc}$. Both numbers are consistent with the result from Giovannini et al. (2011).

The large extent and morphology make it unlikely that the diffuse sources is directly related to the tailed radio galaxy. Giovannini et al. (2011) classify the source as a radio halo. They note that the radio emission permeates both galaxy clumps. However, the possibility that the source is a radio relic projected onto central region of the cluster should also be considered. The source has a very patchy morphology unlike typical radio halos for which the surface brightness follows that of the X-ray emission. Currently we have too little constraints to completely rule out the relic scenario. Deep X-ray observations will be needed to characterize the dynamical state of the cluster. Polarization observations can be used to distinguish between the radio halo and relic scenarios as radio relics are usually strongly polarized (at the 10-20\% level or more) while halos are mostly unpolarized.

\subsection{Abell 697}

Abell 697 is a massive Bautz-Morgan (B/M) type II-III cluster, located at $z=0.282$, with a high X-ray luminosity of $L_{\mathrm{X}, 0.1-2.4 \mathrm{keV}}=19.42 \times 10^{44} \mathrm{erg} \mathrm{s}^{-1}$ (Popesso et al. 2004). The ICM has an elliptical shape and an overall temperature of $8.8_{-0.6}^{+0.7} \mathrm{keV}$ determined from Chandra observations by Maughan et al. (2008). Cavagnolo et al. (2009) reported a slightly higher temperature of $9.52 \mathrm{keV}$. Girardi et al. (2006) found a velocity dispersion of $1334_{-96}^{+114} \mathrm{~km} \mathrm{~s}^{-1}$ for the cluster. They noted this is expected in the case of energy-density equipartition between galaxies and gas. They suggested that the cluster has undergone a complex cluster merger event, occurring mainly along the LOS, with a transverse component in the SSE-NNW direction.

Kempner \& Sarazin (2001) first suggested the presence of diffuse radio emission in the cluster. Venturi et al. (2008) showed the presence of a radio halo using $610 \mathrm{MHz}$ GMRT observations, the halo was also reported by Rudnick \& Lemmerman (2009) and Giovannini et al. (2009). Macario et al. (2010) presented a more detailed study which included GMRT $325 \mathrm{MHz}$ observations. They found the radio halo to have a an ultra-steep spectrum $\left(\alpha_{325 \mathrm{MHz}}^{1.4 \mathrm{GHz}}\right)$ with $\alpha$ about -1.7 to -1.8 .

We detect the radio halo at both 1382 and $1714 \mathrm{MHz}$ with better SNR than the previous observations at $1.4 \mathrm{GHz}$. In our $1382 \mathrm{MHz}$ image we find a total extent of about $750 \mathrm{kpc}$ for the radio halo which is lower than the $1.3 \mathrm{Mpc}$ reported by Macario et al. (2010) at $325 \mathrm{MHz}$. The lower $1382 \mathrm{MHz}$ extent is expected due to the steep radio spectrum of the halo. To better image the diffuse emission and remove the contribution from compact sources, we made images with uniform weighting and excluded data $<2.5 \mathrm{k} \lambda$ at both 1382 and $1714 \mathrm{MHz}$. We then subtracted the clean components of the compact sources from the uv-data before re-imaging. To increase the SNR we combined the 21 and $18 \mathrm{~cm}$ images after convolving them to a common resolution of $29^{\prime \prime} \times 17^{\prime \prime}$, see Fig. 9 . 
R. J. van Weeren et al.: Radio continuum observations of new radio halos and relics from the NVSS and WENSS surveys
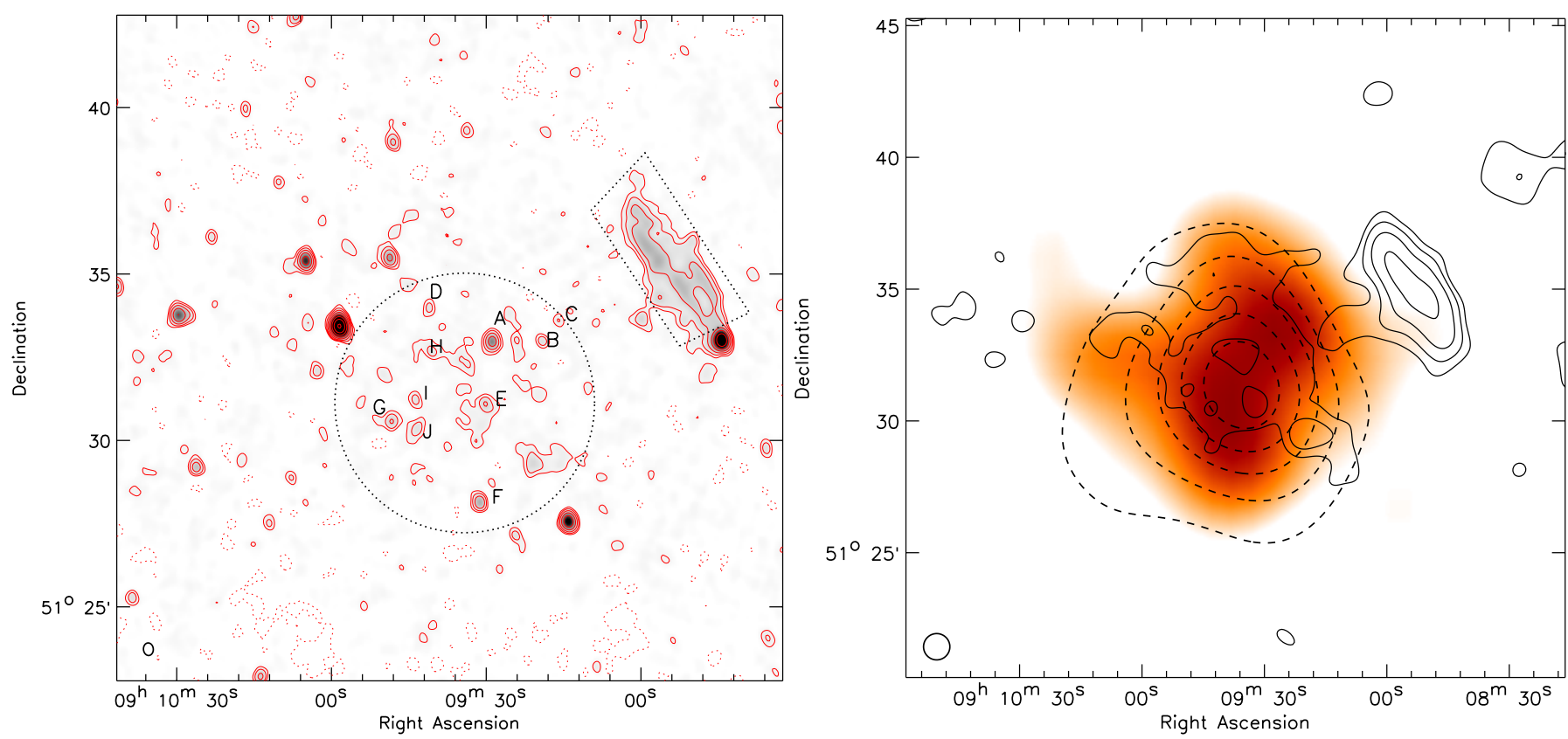

Fig. 5. Left: WSRT $1382 \mathrm{MHz}$ image of A746. Contour levels are drawn as in Fig. 1. Black dotted lines indicate the integration areas for the flux measurements. Discrete sources embedded in the diffuse emission are alphabetically labeled, see Table 3. Right: A746 X-ray emission from ROSAT in orange. The original image from the ROSAT All Sky Survey was convolved with a 225" FWHM Gaussian. Solid contours are from the WSRT $1382 \mathrm{MHz}$ natural weighted image convolved to a resolution of $60^{\prime \prime}$. Compact sources were subtracted and contours are drawn at levels of $[1,2,4,8, \ldots] \times 0.4 \mathrm{mJy}_{\text {beam }^{-1}}$. Dashed contours show the galaxy iso-density distribution derived from the SDSS survey. Contours are drawn at $[1.0,1.4,1.8, \ldots] \times 0.6$ galaxies $\operatorname{arcmin}^{-2}$ selecting only galaxies with $0.16<z_{\text {phot }}<0.29$.
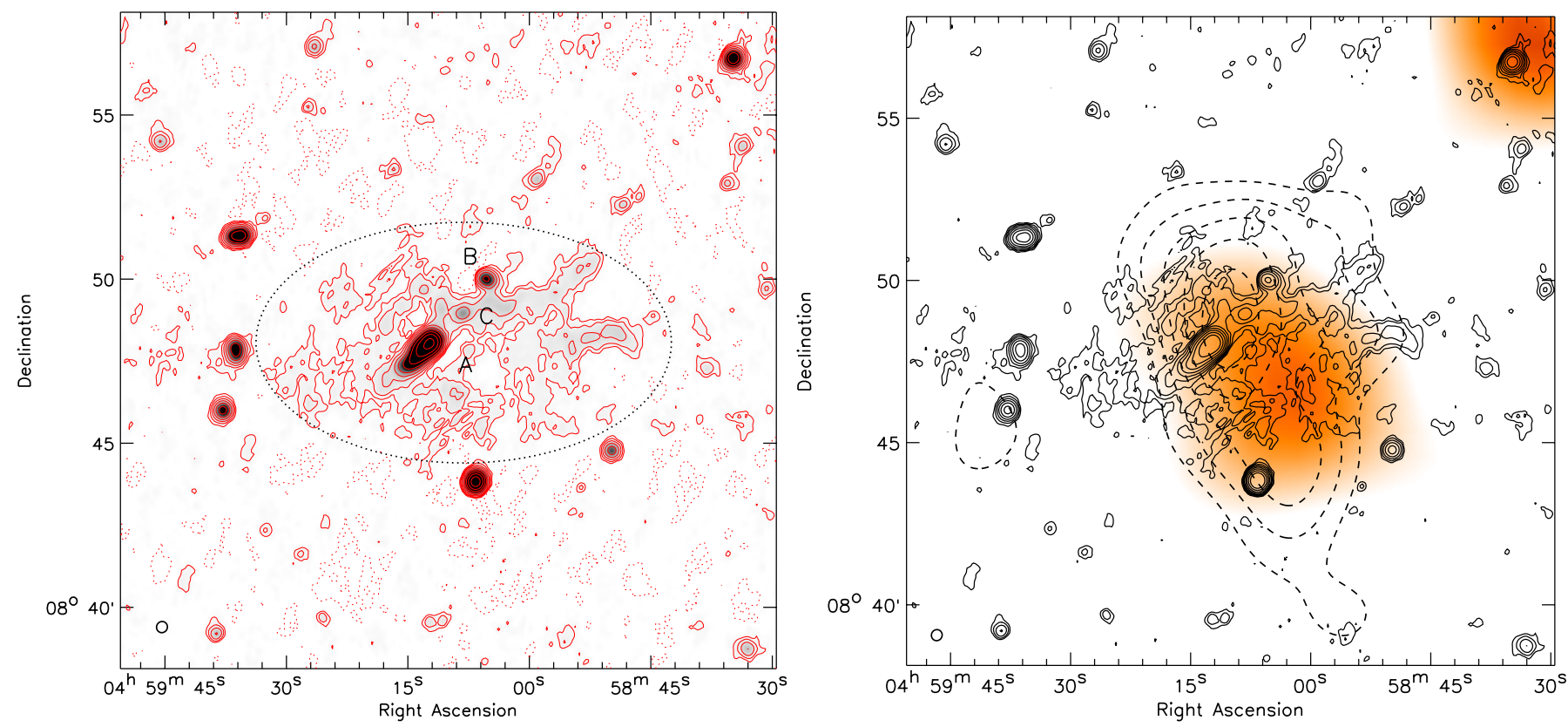

Fig. 6. Left: A523 VLA $1.4 \mathrm{GHz}$ image. Contour levels are drawn as in Fig. 1. Black dotted lines indicate the integration area for the flux measurement. Discrete sources embedded in the diffuse emission are alphabetically labeled, see Table 3. Right: A523 X-ray emission from in orange. The original image from the ROSAT All Sky Survey was convolved with a 225" FWHM Gaussian. Solid contours are from the VLA $1425 \mathrm{MHz}$ image and drawn at levels of $[1,2,4,8, \ldots] \times 4 \sigma_{\mathrm{rms}}$. Dashed contours show the galaxy iso-density distribution derived from INT images. Contours are drawn at $[1.0,1.2,1.4, \ldots] \times 1.6$ galaxies $\operatorname{arcmin}^{-2}$ selecting only galaxies with colors $1.12<V-R<1.42,0.25<R-I<0.55$, i.e., within 0.15 magnitudes the $V-R$ and $R-I$ color of the central cD galaxy.

The discrete sources D, E, and F (see Fig. 9) were not detected with a $S N R>3$. By using the positions from the $1382 \mathrm{MHz}$ image, we could still get an approximate estimate of their fluxes. We subtracted the flux from the discrete sources (Table 3) and took the fluxes reported by Macario et al. (2010) to fit a power-law radio spectrum through the flux measurement with $\alpha=-1.64 \pm 0.06$, see Fig. 8. This confirms that the radio halo has a very steep spectral index, although it is marginally flatter than the -1.7 to -1.8 reported by Macario et al. (2010). 


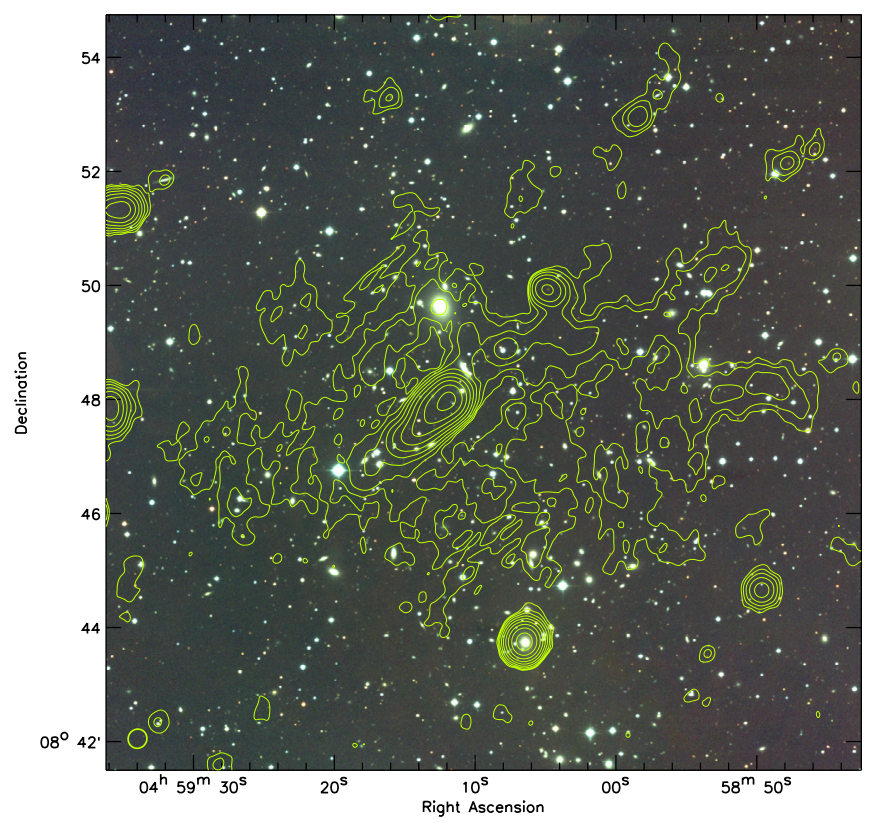

Fig. 7. INT $V, R, I$ color image of Abell 523. Overlaid are the radio contours from Fig. 6.

We do not detect any polarized flux from the radio halo in our WSRT observations. We set an upper limit on the radio halo polarization fraction of $6 \%$ at $1382 \mathrm{MHz}$.

\subsection{Abell 2061}

Abell 2061 is a Bautz-Morgan (B/M) type III cluster located at $z=0.0784$. The cluster has a X-ray luminosity of $L_{\mathrm{X}, 0.1-2.4 \mathrm{keV}}=3.95 \times 10^{44} \mathrm{erg} \mathrm{s}^{-1}$ (Ebeling et al. 1998). The cluster has a velocity dispersion of $673_{-40}^{+49} \mathrm{~km} \mathrm{~s}^{-1}$ (Oegerle $\&$ Hill 2001). The ROSAT image shows the cluster to be extended in the northeast-southwest direction, see Fig. 10. Previous studies report that the cluster is part of the Corona Borealis supercluster (Small et al. 1998; Marini et al. 2004). The galaxy iso-density contours display three substructures located roughly along a northeast southwest axis.

Abell 2067 is located at a projected distance of only $1.8 \mathrm{~h}^{-1} \mathrm{Mpc}$ north from Abell 2061, and the cluster's systematic velocities are separated by $\sim 1600 \mathrm{~km} \mathrm{~s}^{-1}$ (Oegerle \& Hill 2001). Therefore, they probably form a bound system (Marini et al. 2004; Rines \& Diaferio 2006) consisting of a massive cluster (A2061) with a smaller in-falling group/cluster (A2067). A2061 also contains an X-ray extension in the direction of A2067 (towards the north-east) which also suggests a dynamical connection between the two systems (Marini et al. 2004). According to Marini et al., the interaction between the clusters is in the phase in which the cores have not yet met and in which the formation of a shock is expected. The global temperature for A2061 is reported to be $4.53_{-0.38}^{+0.48} \mathrm{keV}$ from BeppoSax observations (Marini et al. 2004). A region with a higher temperature is found in the northern part of A2061 with a temperature of $10.67_{-2.47}^{+3.90} \mathrm{keV}$. This region could correspond to the presence of an internal shock.

Kempner \& Sarazin (2001) reported a possible relic in the southwest periphery of A2061 in WENSS images. They found a flux of $104 \pm 15$ and $19 \pm 3 \mathrm{mJy}$ at $327 \mathrm{MHz}$ and $1.4 \mathrm{GHz}$, respectively. This would give a spectral index of $\alpha=-1.17 \pm$ 0.23. Rudnick \& Lemmerman (2009) also listed the presence of the diffuse peripheral source and measured a flux of $120 \mathrm{mJy}$ in the WENSS image. They also claimed the presence of additional diffuse emission in the center of the cluster which could make up a radio halo.

With our WSRT observations we confirm the presence of the diffuse radio source in the southwestern periphery of A2061 and we also classify it as radio relic. We cannot confirm the presence of a radio halo. The radio relic is clearly seen in both the 21 and $18 \mathrm{~cm}$ WSRT images, Fig. 11. The relic is located at a distance of $1.5 \mathrm{Mpc}$ from the cluster center and has a largest angular extent of $7.7^{\prime}$, corresponding to a physical size of $675 \mathrm{kpc}$. In the direction towards the cluster center the relic has an extent of $\sim 320 \mathrm{kpc}$. The western outer boundary of the relic is somewhat more pronounced, while the emission fades more slowly inwards to the cluster center. The relic consist of a northern and fainter southern component. Two compact sources are found directly to the north and south of the relic.

An optical color image at the location of the relic is shown in Fig. 13. This image does not reveal any obvious counterparts to the radio relic. One galaxy is located at the brightest region of the relic, but no compact radio source is associated with that galaxy. The bright compact sources to the north and south of the relic are both associated with background galaxies unrelated to A2061 because of their small angular sizes $\left(\$ 3^{\prime \prime}\right)$ and very red color. For comparison, we have marked two galaxies located approximately at the distance of A2061 (Fig. 13).

The radio spectrum is fitted by a single power-law spectrum, see Fig. 12. We find $\alpha=-1.03 \pm 0.09$. The $325 \mathrm{MHz}$ WENSS flux is from Rudnick \& Lemmerman. The $325 \mathrm{MHz}$ measurement is however uncertain as the SNR on the relic is low. To better constrain the spectral index an additional low-frequency flux measurement is needed.

The picture that emerges from our observations is that of a radio relic tracing a shock wave from a cluster merger event. The merger event does not seem to be directly related to the cluster A2067, located north of A2061. Instead the shock wave is more likely related to the observed substructures seen in the SDSS galaxy iso-density contours.

\subsection{Abell 3365}

Abell 3365 (Abell et al. 1989) is located at a redshift of $z=$ 0.0926 (Struble \& Rood 1999). The cluster is listed as a BautzMorgan (B/M) type II cluster. The ESO Nearby Abell Cluster Survey (ENACS, Katgert et al. 1996) measured a velocity dispersion of $1153 \mathrm{~km} \mathrm{~s}^{-1}$ (Mazure et al. 1996) for A3365, quite high compared to other clusters in the sample. Galaxy cluster RXC J0548.8-2154 is located 9' to the west of the NED listed position for A3365 at a redshift of $z=0.0928$. The galaxy distribution, see Fig. 14 (right panel), peaks at the center of A3365, while the X-ray peak is located at the position of RXC J0548.82154. The galaxy distribution around A3365 is complex, with two main concentrations along an east-west axis and a smaller concentration at the far west. The ICM distribution is also complex and elongated in the east-west direction. Based on the very similar redshift, and complex X-ray emission and galaxy distribution, we conclude that A3365 and RXC J0548.8-2154 belong to the same merging system to which we will simply refer as A3365.

In the NVSS image we noted the presence of a peripheral elongated radio source to the east of the cluster center. The WSRT image (Fig. 14, left panel) reveals a second smaller 

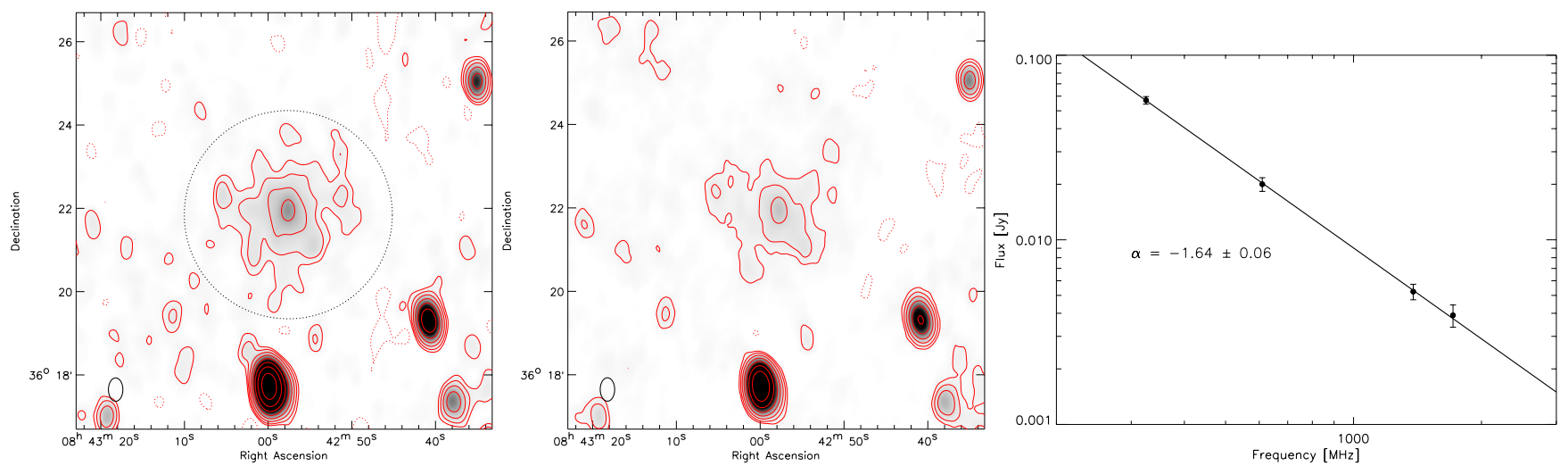

Fig. 8. Left: WSRT $1382 \mathrm{MHz}$ image of Abel 697. Contour levels are drawn as in Fig. 1. Black dotted lines indicate the integration area for the flux measurements. Middle: WSRT $1714 \mathrm{MHz}$ image of Abell 697. Contour levels are drawn as in Fig. 1. Right: A697 radio halo spectrum. Flux measurements at 325 and $610 \mathrm{MHz}$ are taken from Macario et al. (2010).

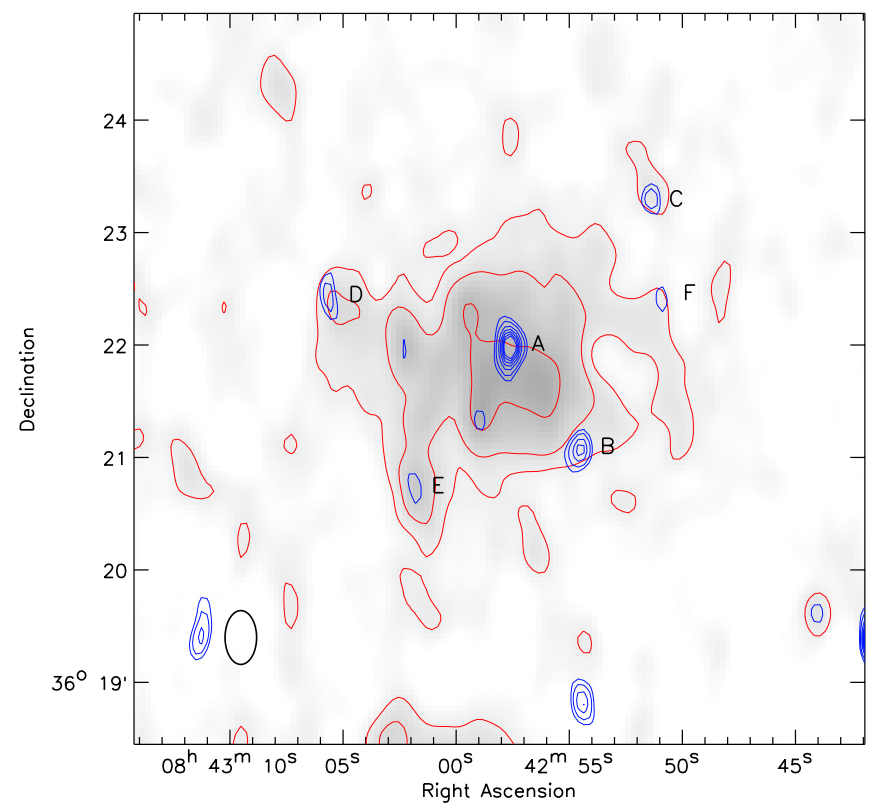

Fig. 9. WSRT combined 1382 and $1714 \mathrm{MHz}$ image of the radio halo in Abel 697, compact sources were subtracted from the uv-data. Red contour levels are drawn at $[1,2,4,8 \ldots] \times 60 \mu \mathrm{Jy}_{\text {beam }}^{-1}$. Blue contours display the compact sources at $1382 \mathrm{MHz}$. This high-resolution image $\left(19^{\prime \prime} \times 10^{\prime \prime}\right)$ was made with uniform weighting and data $<2.5 \mathrm{k} \lambda$ was excluded. Contour levels are drawn at $\sqrt{[1,2,4,8 \ldots]} \times 100 \mu \mathrm{Jy}_{\text {beam }}^{-1}$. Discrete sources embedded in the diffuse emission are alphabetically labeled, see Table 3.

diffuse source on the west side of the cluster. The diffuse sources are also detected in the VLA images (Fig. 15). For both sources we cannot identify obvious optical counterparts in our INT images (Fig. 16). The east and western sources have an angular extent of $5.5^{\prime}$ and $2.3^{\prime}$, which correspond to 560 and $235 \mathrm{kpc}$ at the distance of A3365. We classify the eastern source as a radio relic. Most likely, the western source is another radio relic, which means that A3365 hosts a double radio relic system, but more observations are needed to confirm this classification. This interpretation is supported by the elongated X-ray and galaxy distribution which suggests a merger event along an east-west axis (Fig. 14 right panel). We also made an image where we

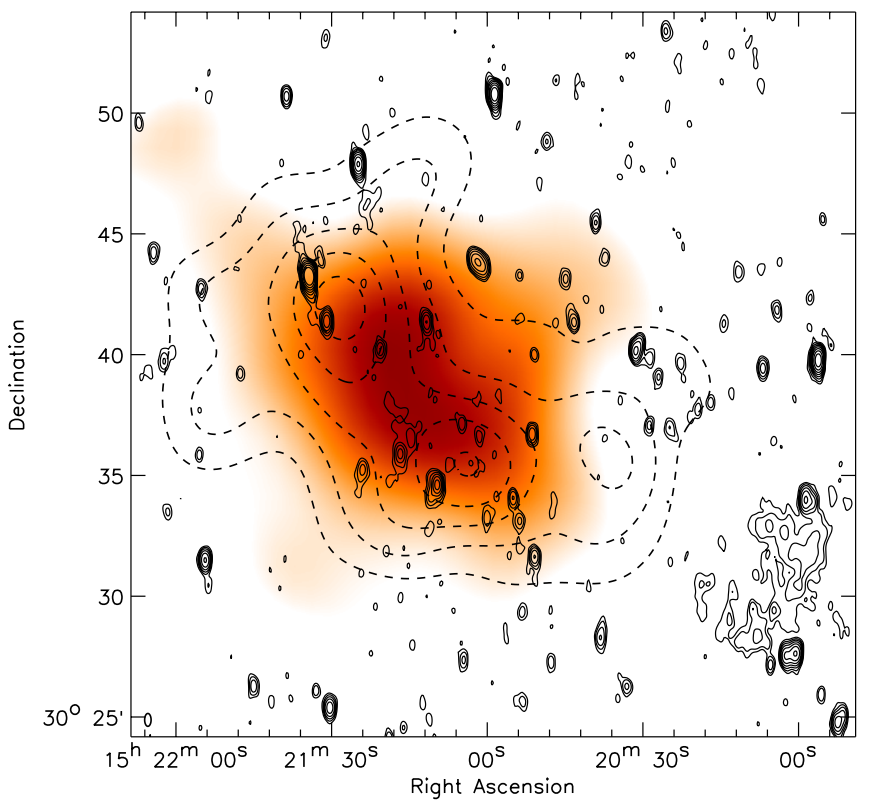

Fig. 10. A2061 X-ray emission from ROSAT in orange. The original image from the ROSAT All Sky Survey was convolved with a 225" FWHM Gaussian. Solid contours are from the WSRT $1382 \mathrm{MHz}$ image and drawn at levels of $[1,2,4,8, \ldots] \times 4 \sigma_{\text {rms }}$. Dashed contours show the galaxy iso-density distribution derived from the SDSS survey. Contours are drawn at $[1.0,1.4,1.8, \ldots] \times 0.55$ galaxies $\operatorname{arcmin}^{-2}$ selecting only galaxies with $0.05<z_{\text {phot }}<0.1$.

subtracted the compact sources from the uv-data. To make this image, we subtracted the clean components from a uniformly weighted image (CnB array), from the DnC array data. This image is overlaid in Fig. 14.

\subsection{CIZA J0649.3+1801}

CIZA J0649.3+1801 is a little studied galaxy cluster located at $z=0.064$ discovered by Ebeling et al. (2002) at a galactic latitude of $b=7.668^{\circ}$. The cluster has a moderate X-ray luminosity of $L_{\mathrm{X}}, 0.1-2.4 \mathrm{keV}=2.38 \times 10^{44} \mathrm{erg} \mathrm{s}^{-1}$. The cluster forms part of a supercluster in the Zone of Avoidance hidden by the Milky Way (Kocevski et al. 2007). 

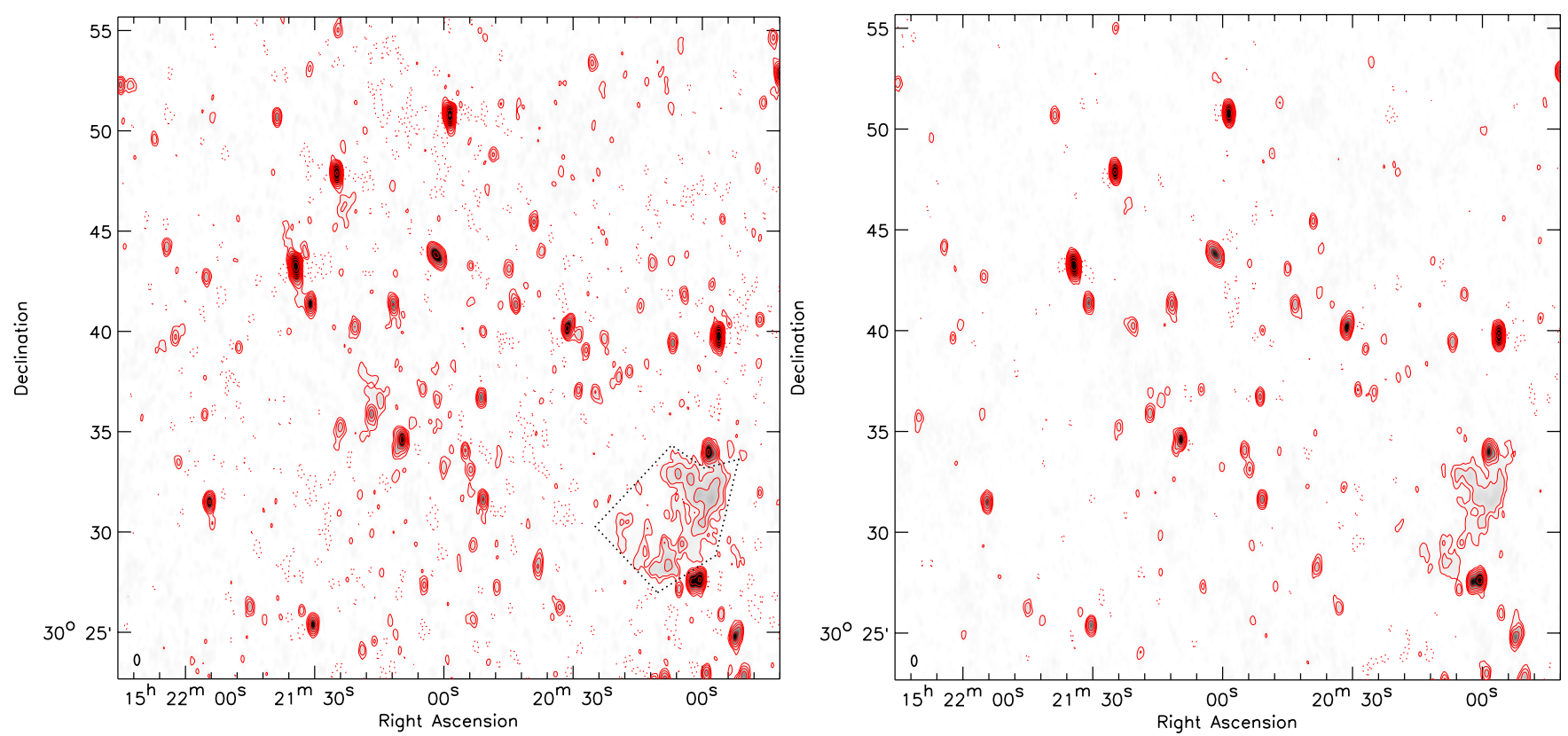

Fig. 11. Left: A2061 WSRT $1382 \mathrm{MHz}$ image. Contour levels are drawn as in Fig. 1. Black dotted lines indicate the integration area for the flux measurements. Right: A2061 WSRT 1714 MHz image. Contour levels are drawn as in Fig. 1.

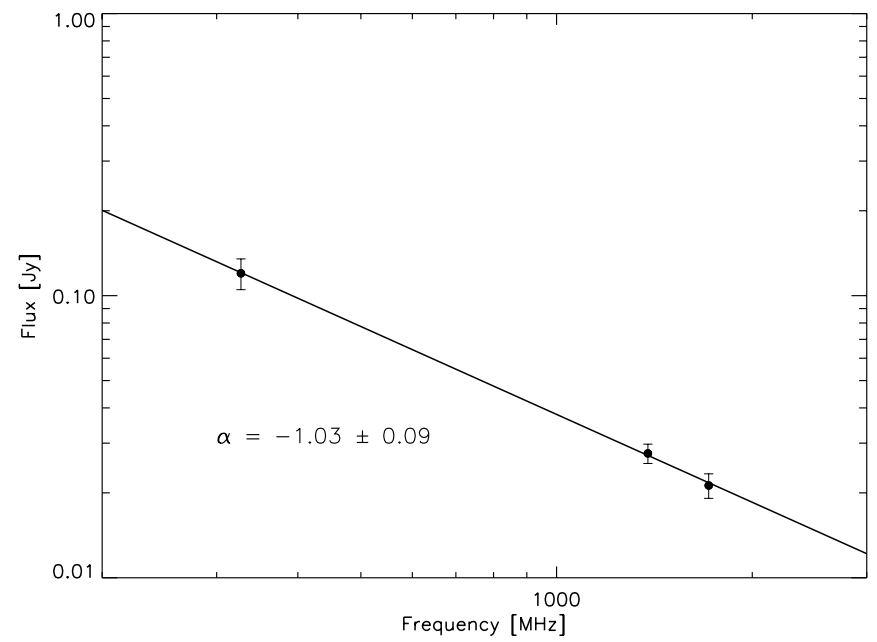

Fig. 12. A2061 radio relic spectrum. Flux measurement at $325 \mathrm{MHz}$ is taken from Rudnick \& Lemmerman (2009).

We discovered the presence of a diffuse $\sim 10^{\prime}$ elongated source to the west of the cluster in the NVSS survey. We do not find an optical counterpart for the source in our WHT images. In the GMRT image, see Fig. 17 (left panel), the source has a total extent of 10.6' which corresponds to a size of about $800 \mathrm{kpc}$ at the distance of CIZA J0649.3+1801. We therefore classify the source as a peripheral radio relic located at $0.8 \mathrm{Mpc}$ from the cluster center. The relic is also visible in the $241 \mathrm{MHz}$ image, although the SNR on the relic is very low (Fig. 17 right panel).

The ROSAT image reveals another fainter X-ray source located between the radio relic and the cluster center. The source is not resolved and therefore it could be unrelated to the cluster. Also we do not detect any group or cluster of galaxies associated with this source in our optical WHT images. Therefore, this X-ray source seems to be unrelated to CIZA J0649.3+1801.

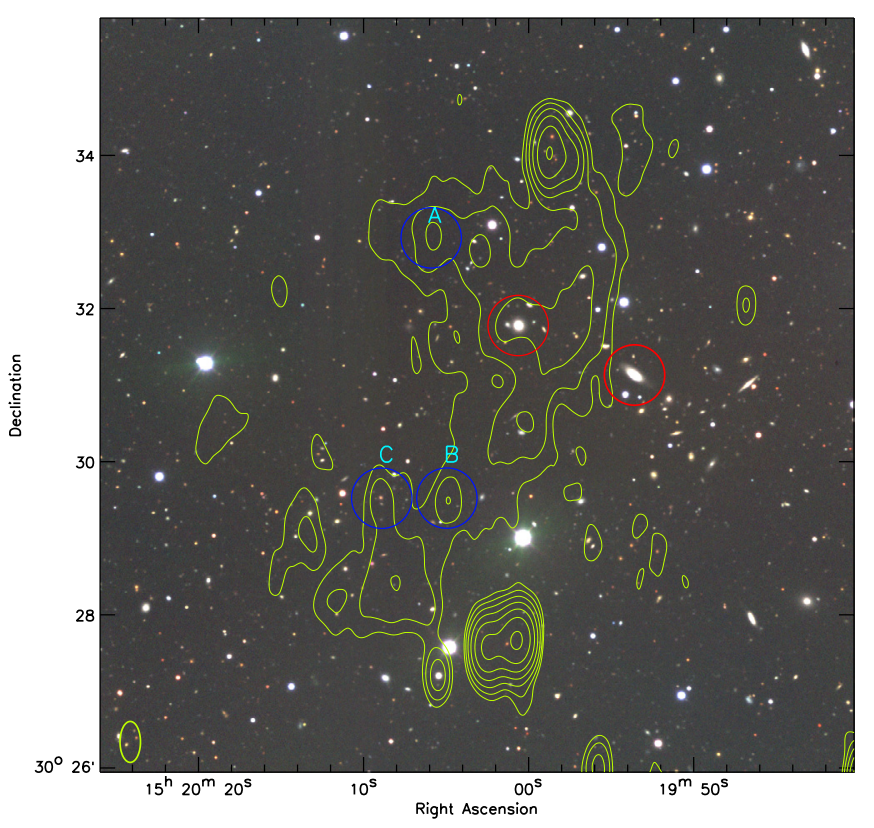

Fig. 13. A2061 optical WHT $V, R, I$ color image at the location of the radio relic. Radio contours are from Fig. 11. The two red circles indicate galaxies with spectroscopic redshifts of 0.0815 (west) and 0.061 (Abazajian et al. 2009). Discrete sources embedded in the diffuse emission are alphabetically labeled, see Table 3.

\subsection{CIZA J0107.7+5408}

CIZA J0107.7+5408 is located at $z=0.1066$ (Crawford et al. $1995)$ and has quite a high X-ray luminosity of $L_{\mathrm{X}}, 0.1-2.4 \mathrm{keV}=$ $5.42 \times 10^{44} \mathrm{erg} \mathrm{s}^{-1}$ (Ebeling et al. 2002). The cluster is projected relatively close to the galactic plane with $b=-8.65^{\circ}$. Both the NVSS and WENSS survey images display an extended diffuse radio source located roughly at the cluster center. Our WSRT image clearly reveals the presence of a somewhat elongated radio 
R. J. van Weeren et al.: Radio continuum observations of new radio halos and relics from the NVSS and WENSS surveys
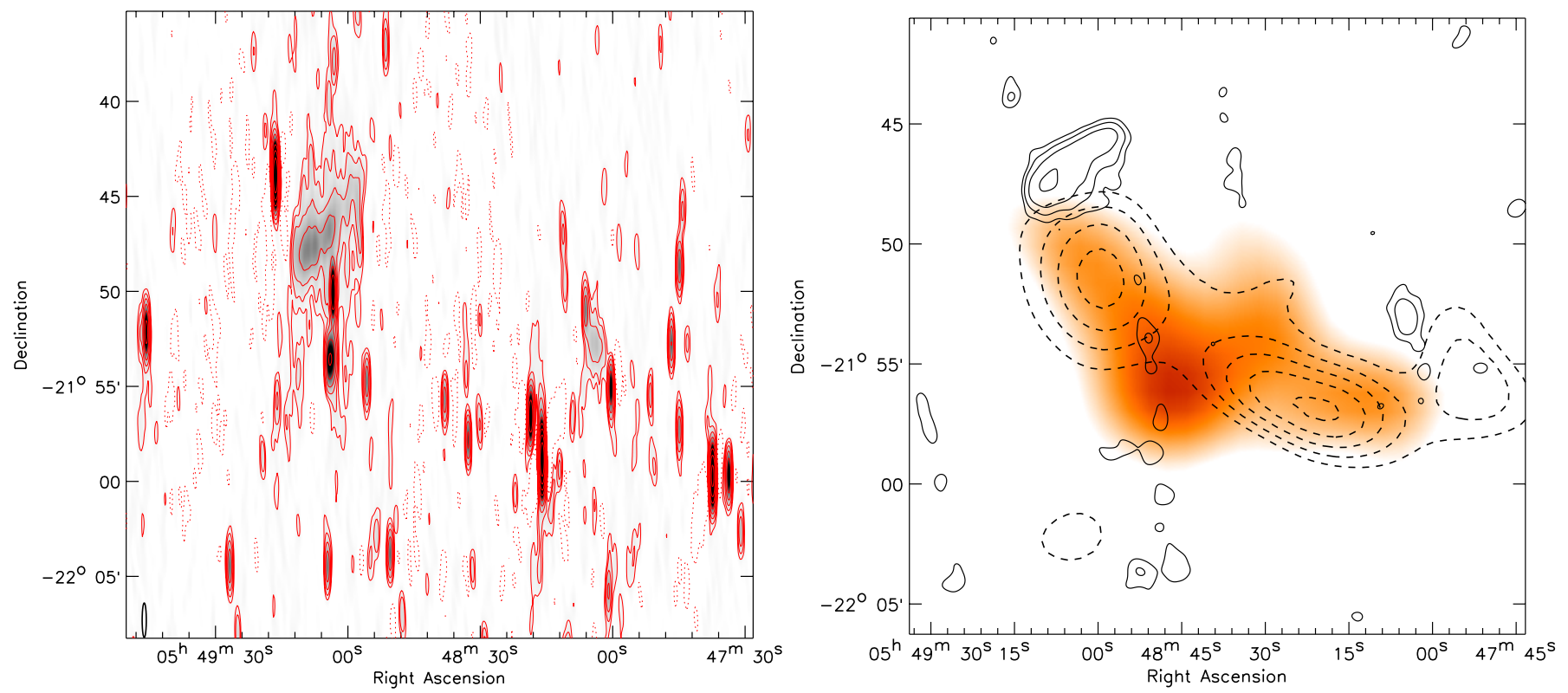

Fig. 14. Left: A3365 WSRT $1382 \mathrm{MHz}$ image. Contour levels are drawn as in Fig. 1. Right: A3365 X-ray emission from ROSAT in orange. The original image from the ROSAT All Sky Survey was convolved with a 225" FWHM Gaussian. Solid contours are from a VLA 1.4 GHz image with compact sources subtracted from the uv-data. This DnC array image has a resolution of $47^{\prime \prime} \times 42^{\prime \prime}$. Contours are drawn at levels of $[1,2,4,8, \ldots] \times 0.4 \mathrm{mJy}^{\text {beam }}{ }^{-1}$. Dashed contours show the galaxy iso-density distribution derived from INT images. Contours are drawn at $[1.0,1.1,1.2, \ldots] \times 0.78$ galaxies arcmin ${ }^{-2}$ selecting only galaxies with colors $0.6<V-R<0.9,0.54<R-I<0.84$, i.e., within 0.15 mag the $V-R$ and $R-I$ color of the largest $\mathrm{cD}$ galaxy.
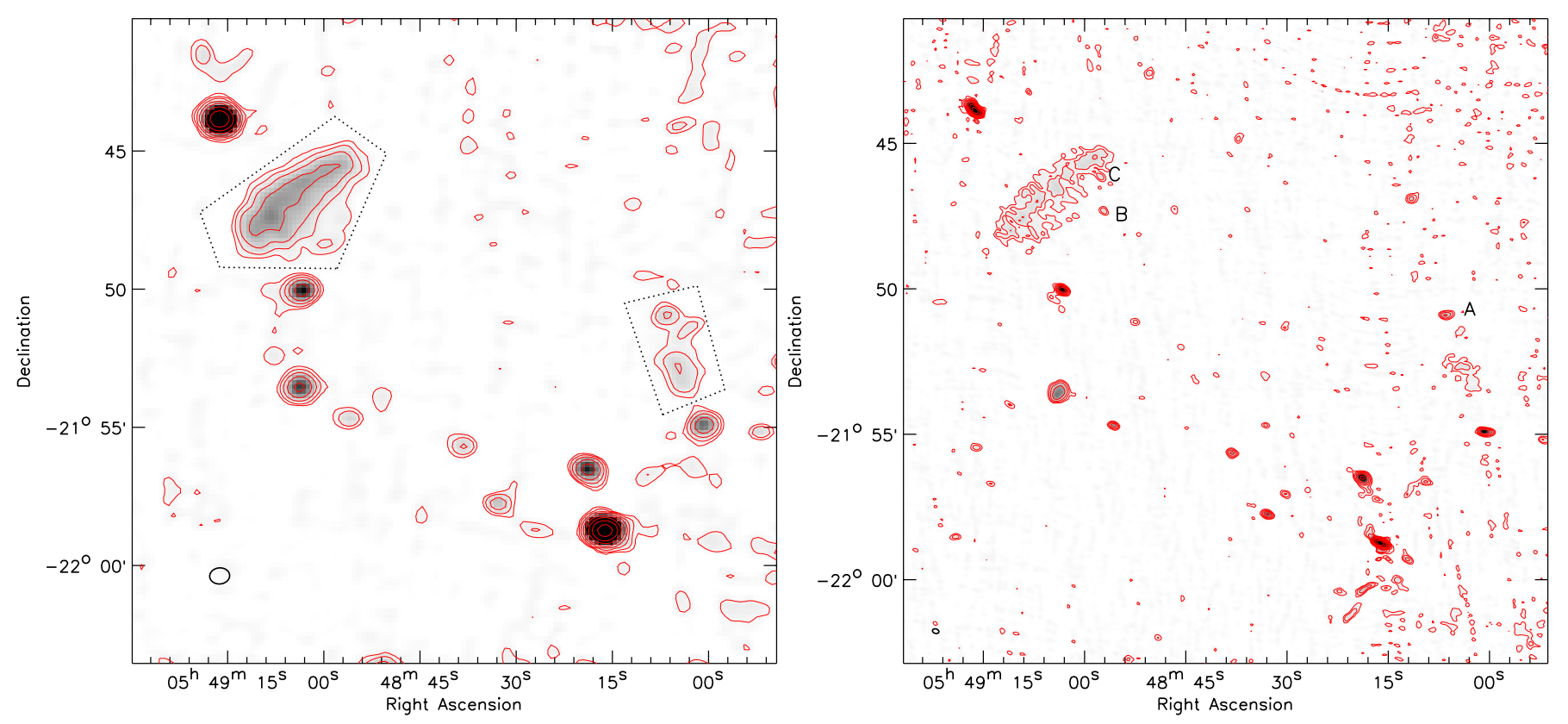

Fig. 15. A3365 VLA $1.4 \mathrm{GHz}$ DnC (left) and CnB (right) array images. Contour levels are drawn as in Fig. 1. Black dotted lines indicate the integration areas for the flux measurements. Discrete sources embedded in the diffuse emission are alphabetically labeled, see Table 3.

halo with a largest extent in the north-south direction of $1.1 \mathrm{Mpc}$. The galaxy and ICM distribution are also elongated along the major axis of the radio halo, see Fig. 18, which supports the scenario that the cluster is currently undergoing a merger event. An image of the radio halo with the discrete sources subtracted (using the same technique as described in Sect. 3.4) is overlaid with contours. The radio power of $3.8 \times 10^{24} \mathrm{~W} \mathrm{~Hz}^{-1}$ is consistent with the $L_{\mathrm{X}}-P_{1.4 \mathrm{GHz}}$ correlation for giant radio halos.

\subsection{Abell 2034}

Abell 2034 is a merging galaxy cluster as revealed by a Chandra X-ray study from Kempner et al. (2003). White (2000) reported a global cluster temperature of $7.6 \mathrm{keV}$ in agreement with the value of $7.9 \pm 0.4 \mathrm{keV}$ found by Kempner et al. (2003). Kempner $\&$ Sarazin (2001) suggested the presence of a radio relic on the basis of a WENSS image. The diffuse emission is located close to the position of a cold front found by Kempner et al. (2003). 

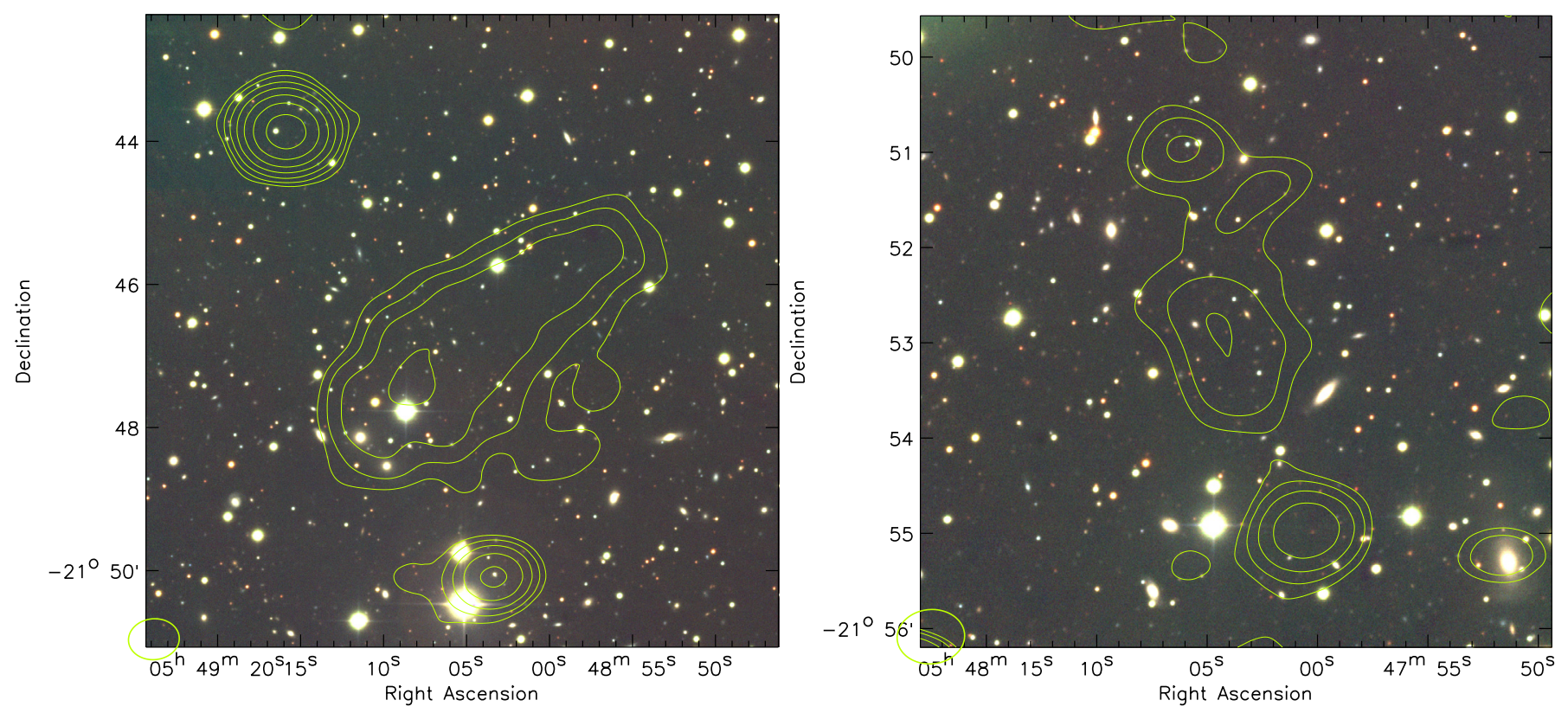

Fig. 16. A3365 optical INT $V, R, I$ color images at the location of the radio relics. Radio contours are from Fig. 15 (right panel).
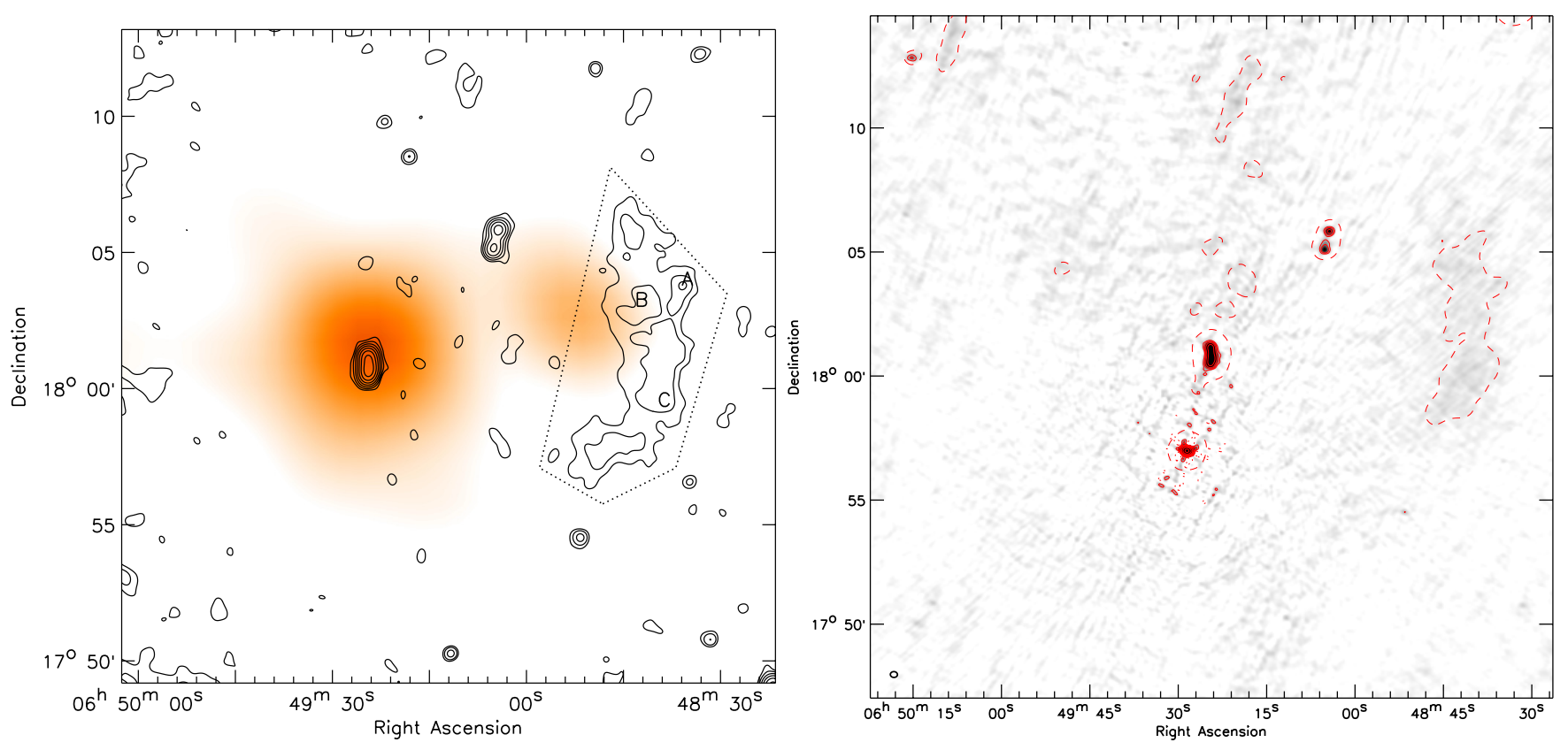

Fig. 17. Left: CIZA J0649.3+1801 X-ray emission from ROSAT in orange. The original image from the ROSAT All Sky Survey was convolved with a $225^{\prime \prime}$ FWHM Gaussian. Solid contours are from the GMRT $610 \mathrm{MHz}$ image convolved to a resolution of 25" and drawn at levels of $[1,2,4,8, \ldots] \times 4 \sigma_{\text {rms. }}$. The source NVSS J064928+175700 was removed using the "peeling"-method (e.g., Noordam 2004). Black dotted lines indicate the integration area for the flux measurement. Discrete sources embedded in the diffuse emission are alphabetically labeled, see Table 3. Right: CIZA J0649.3+1801 GMRT $241 \mathrm{MHz}$ image. Contour levels are drawn as in Fig. 1 . The dashed line is the $15 \mathrm{mJy}^{\mathrm{beam}}{ }^{-1}$ contour of the $241 \mathrm{MHz}$ images convolved to a circular beam of $45^{\prime \prime}$.

The presence of diffuse emission was confirmed by Giovannini et al. (2009) and was classified as an irregular elongated radio halo.

We also observe the diffuse emission to brighten at the position of the cold front in the WSRT image. The SDSS galaxy distribution (Fig. 19, right panel) is bimodal which supports the scenario that the cluster is undergoing a merger event. We consider the classification of the source uncertain, since the radio emission does not show a very clear correlation with the X-rays. It could therefore also be a radio relic. The radio power $\left(P_{1.4 \mathrm{GHz}}=0.28 \times 10^{24} \mathrm{~W} \mathrm{~Hz}^{-1}\right)$ though is in agreement with the $L_{\mathrm{X}}-P_{1.4 \mathrm{GHz}}$ correlation for giant radio halos (e.g., Liang et al. 2000; Cassano et al. 2006). The total flux we find is $7.3 \pm 2.0 \mathrm{mJy}$, lower than the $13.6 \pm 1.0 \mathrm{mJy}$ reported by Giovannini et al. (2009). The reason for the difference is unclear because Giovannini et al. (2009) do not report the integration area and the fluxes of the discrete sources that were subtracted. Deeper observations which sufficient resolution are needed to 
R. J. van Weeren et al.: Radio continuum observations of new radio halos and relics from the NVSS and WENSS surveys
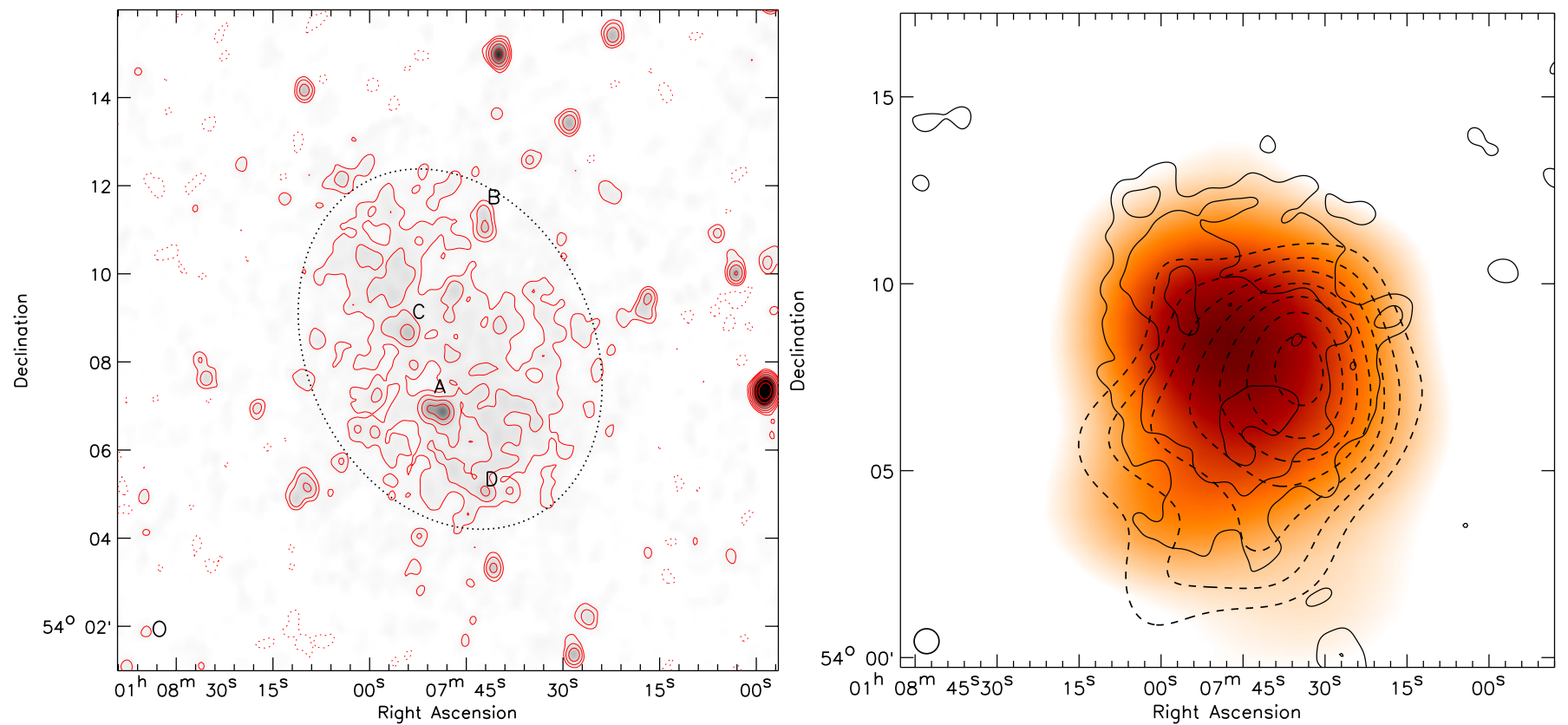

Fig. 18. Left: CIZA J0107.7+5408 WSRT $1382 \mathrm{MHz}$ image. Contour levels are drawn as in Fig. 1. Black dotted lines indicate the integration area for the flux measurement. Discrete sources embedded in the diffuse emission are alphabetically labeled, see Table 3. Right: CIZA J0107.7+5408 X-ray emission from ROSAT in orange. The original image from the ROSAT All Sky Survey was convolved with a 225" FWHM Gaussian. Solid contours are from the WSRT $1382 \mathrm{MHz}$ image convolved to a resolution of 40". Compact sources were subtracted and contours are drawn at levels of $[1,2,4,8, \ldots] \times 0.3 \mathrm{mJy}_{\text {beam }}{ }^{-1}$. Dashed contours show the galaxy iso-density distribution derived from INT images. Contours are drawn at $[1.0,1.2,1.4, \ldots] \times 0.3$ galaxies $\operatorname{arcmin}^{-2}$ selecting only galaxies with colors $0.85<V-R<1.15,0.6<R-I<0.9$, i.e., within 0.15 mag the $V-R$ and $R-I$ color of the central $\mathrm{cD}$ galaxy.
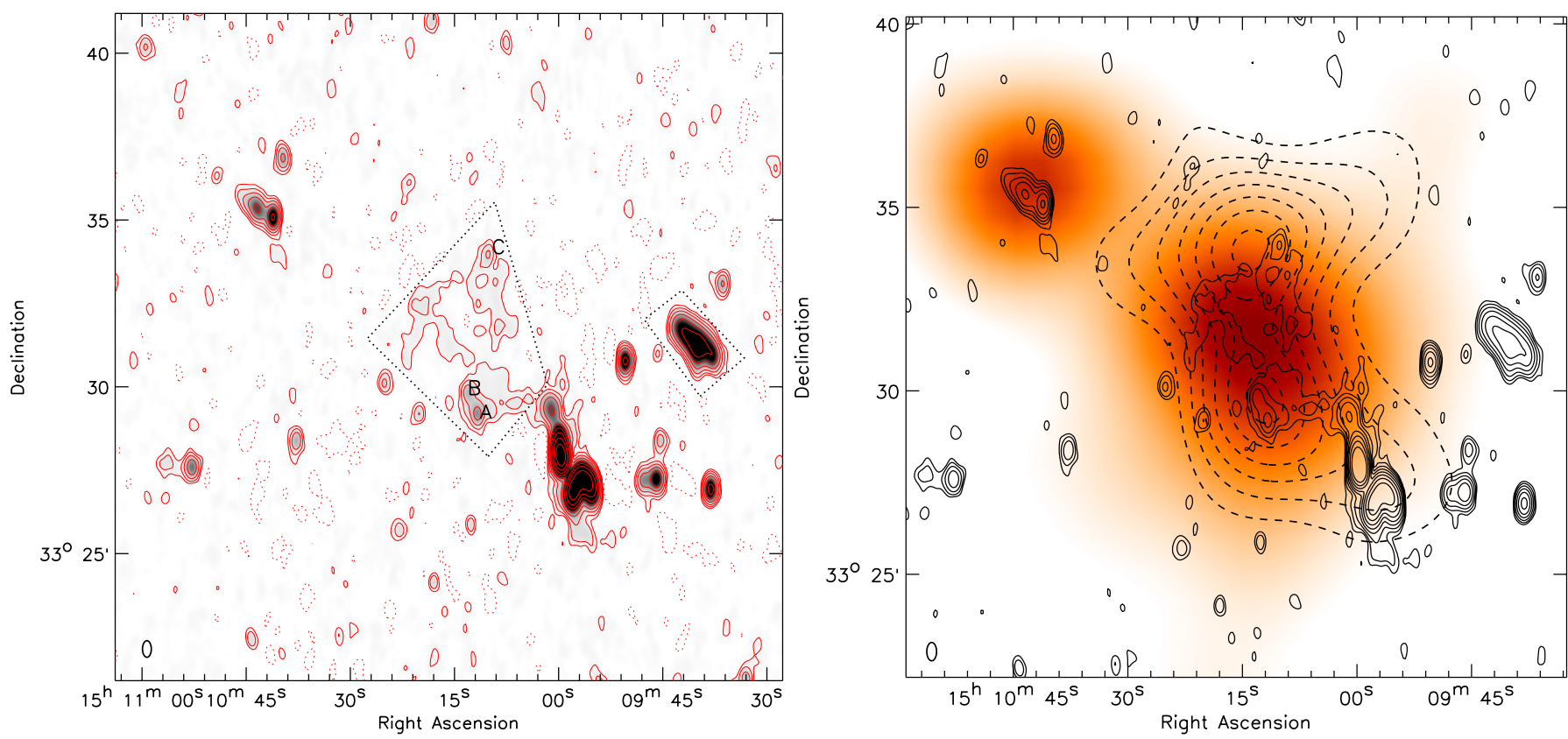

Fig. 19. Left: A2034 WSRT $1382 \mathrm{MHz}$ image. Contour levels are drawn as in Fig. 1. Black dotted lines indicate the integration areas for the flux measurements. Discrete sources embedded in the diffuse emission are alphabetically labeled, see Table 3. Right: A2034 X-ray emission from ROSAT in orange. The original image from the ROSAT All Sky Survey was convolved with a 180" FWHM Gaussian. Solid contours are from the WSRT $1382 \mathrm{MHz}$ image and drawn at levels of $[1,2,4,8, \ldots] \times 4 \sigma_{\mathrm{rms}}$. Dashed contours show the galaxy iso-density distribution derived from the SDSS survey. Contours are drawn at $[1.0,1.2,1.4, \ldots] \times 1.1$ galaxies $\operatorname{arcmin}^{-2}$ selecting only galaxies with $0.07<Z_{\text {phot }}<0.16$.

classify the nature of the diffuse emission. Polarimetric observations could be very helpful here as radio relics are usually highly polarized.

We report the detection of a new relatively small radio relic located west of the cluster center (NVSS J150940+333119,
WN B1507.6+3342, 7C 150739.39+334252.00). This relic is already visible in the previous studies mentioned above but was not recognized as a radio relic probably because it is quite compact. The source has a size about 220 by $75 \mathrm{kpc}$ and a spectral index $\approx-1.2$, including flux measurements from the NVSS, 


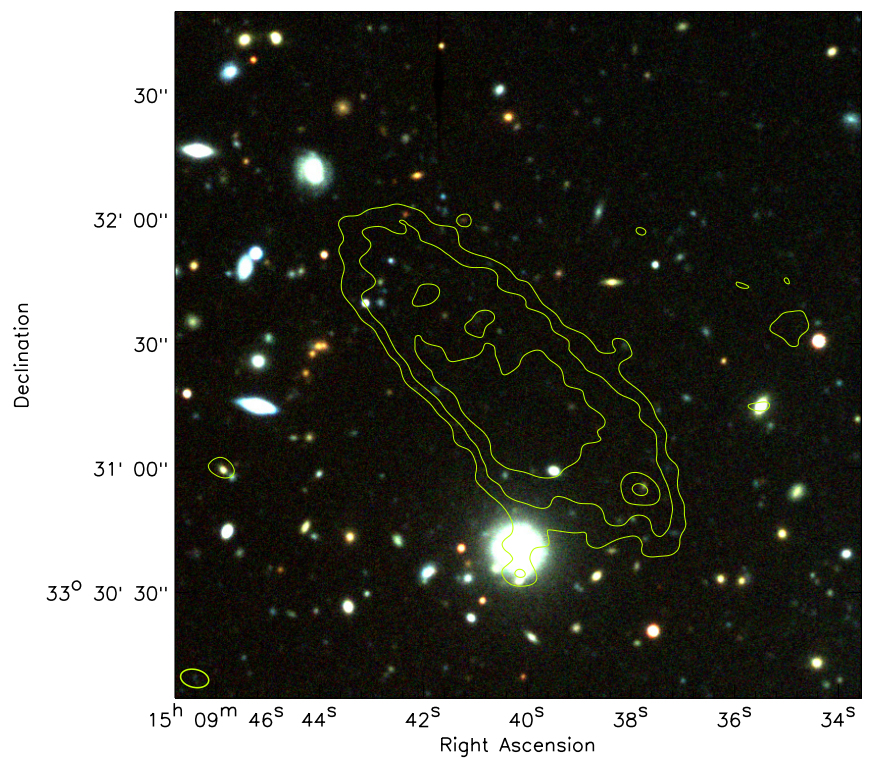

Fig. 20. A2034 WHT $V, R, I$ color image around the small radio relic. Contours at $610 \mathrm{MHz}$ from the GMRT are overlaid and drawn at levels of $[1,2,4,8, \ldots] \times 4 \sigma_{\text {rms }}$. The noise level in the image is $41 \mu \mathrm{Jy} \mathrm{beam}^{-1}$ and the beam size is $6.8^{\prime \prime} \times 4.3^{\prime \prime}$.

WENSS and 7C (Hales et al. 2007) surveys . A high-resolution $610 \mathrm{MHz}$ GMRT of the relic overlaid on an optical WHT image is shown in Fig. 20. The source does not have an optical counterpart which should have easily been visible in the WHT image. The radio plasma could have originated from the compact radio source located immediately south of the relic. On the other hand the spectral index is more typical of a relic directly tracing a shock wave.

\subsection{0. $R X C J 1053.7+5452$}

RXC J1053.7+5452 is located at $z=0.0704$. Aguerri et al. (2007) reported a velocity dispersion of $665_{-45}^{+51} \mathrm{~km} \mathrm{~s}^{-1}$ and an $r_{200}$ radius of $1.52 \mathrm{Mpc}$ based on SDSS data. Rudnick \& Lemmerman (2009) mentioned the presence of a diffuse radio relic with an extent of about $1 \mathrm{Mpc}$ on the west side of the cluster. Rudnick \& Lemmerman reported a total flux of $0.36 \mathrm{Jy}$ at $325 \mathrm{MHz}$. The low-surface brightness radio relic is also visible in the WSRT image. We find a total extent of $600 \mathrm{kpc}$, lower than that of Rudnick \& Lemmerman (2009). The ROSAT image overlaid with SDSS galaxy iso-density contours and the 1382 MHZ WSRT image are shown in Fig. 21. The galaxy distribution seems irregular with the main peak to the southeast of the $\mathrm{X}$-ray center and a second peak to the northwest. The radio relic is roughly located along a line connecting these two galaxy concentrations. This is expected for a cluster merger event, with the shock waves traveling outwards along the merger axis. Deeper radio observations are necessary to better map the extent of this faint radio relic.

\section{Discussion}

Giant radio relics are proposed to directly trace merger shock waves in galaxy clusters. Simulations show that in the case of a binary merger event two shell-like shock waves form at the moment of core passage (e.g., Roettiger et al. 1999; Ricker \& Sarazin 2001). These shock waves then move outwards into the lower density ICM of the cluster's outskirts. Double radio relics are thought to trace these binary merger events, with two symmetric shock waves on each side of the cluster center. However, often merger events are more complex with multiple substructures merging, so that relics are not necessarily symmetric structures and not always come in pairs. Also, the shock structures may break up when they interact with the galaxy filaments connected to the cluster (e.g., Paul et al. 2011). Still, it is expected that relics are mainly found along the main axis of a merger event, while their orientation is perpendicular to this axis.

We have tested this prediction by constructing a sample of 35 relics, taking relics from the literature and those presented in this paper (see Table 4). We did not include any radio relics classified as AGN relics or radio phoenices and selected only relics in clusters which are detected in the ROSAT All Sky Survey (RASS) images.

For every relic we record the end positions of their largest spatial extent (R1, R2), see Fig. 22. The line connecting these two points we define as the relic's major axis. The midpoint between the two extrema we take as a the relic's center position. The RASS image can be used to estimate the position of a possible merger axis. We convolve the ROSAT X-ray images for each cluster to the same spatial resolution of $650 \mathrm{kpc}$. We then fit a 2-dimensional elliptical Gaussian to the X-ray emission. We record both the position of the major axis and the center of the fitted Gaussian. For the merger axis we take as a proxy the major axis of the fitted X-ray emission. Finally, we compute the angle $\left(\alpha_{\mathrm{r}}\right)$ between the major axis of the ICM and the line cluster center-radio relic center. The resulting histogram is shown in Fig. 22. From this histogram we see that relics are preferably found along the major axis of the ICM. This is in line with the simple picture that shock waves propagate outwards along the merger axis. We also calculate the angle $(\beta)$ between the major axis of the ICM and the relic's major axis. We find that most relics are oriented perpendicular to the ICM major axis, also in agreement with a proposed shock origin for radio relics.

\subsection{Comparison with the REFLEX and NORAS X-ray clusters}

We also compared the properties of cluster hosting radio relics with the X-ray clusters from the NORAS (Böhringer et al. 2000) and REFLEX (Böhringer et al. 2004) surveys. The NORAS survey contains 378 galaxy clusters and has an estimated completeness of about $50 \%$ at an X-ray Flux of $F_{\mathrm{X}}, 0.1-2.4 \mathrm{keV}=$ $3.0 \times 10^{-12} \mathrm{erg} \mathrm{s}^{-1} \mathrm{~cm}^{-2}$. The REFLEX sample contains 447 galaxy clusters and has and a completeness of about $90 \%$ at the same flux level as the NORAS survey. For each cluster in this sample, we fitted a 2-dimensional elliptical Gaussian to the $\mathrm{X}$-ray emission from the RASS image, using the same procedure as for the clusters hosting giant relics. For some clusters the fitting procedure did not converge because of nearby bright confusing X-ray sources, these cluster were not included in the analysis. The resulting histogram is displayed in Fig. 23. The distribution of the major-minor axis ratios for the relic cluster sample is broader. This is expected since relics should be found in merging cluster which are typically more elongated.

In addition, we compare both the X-ray luminosity and redshift distribution of the NORAS-REFLEX sample with the relic sample. We selected all clusters with a flux larger than $3.0 \times 10^{-12} \mathrm{erg} \mathrm{s}^{-1} \mathrm{~cm}^{-2}$ in the ROSAT band. Below this flux 

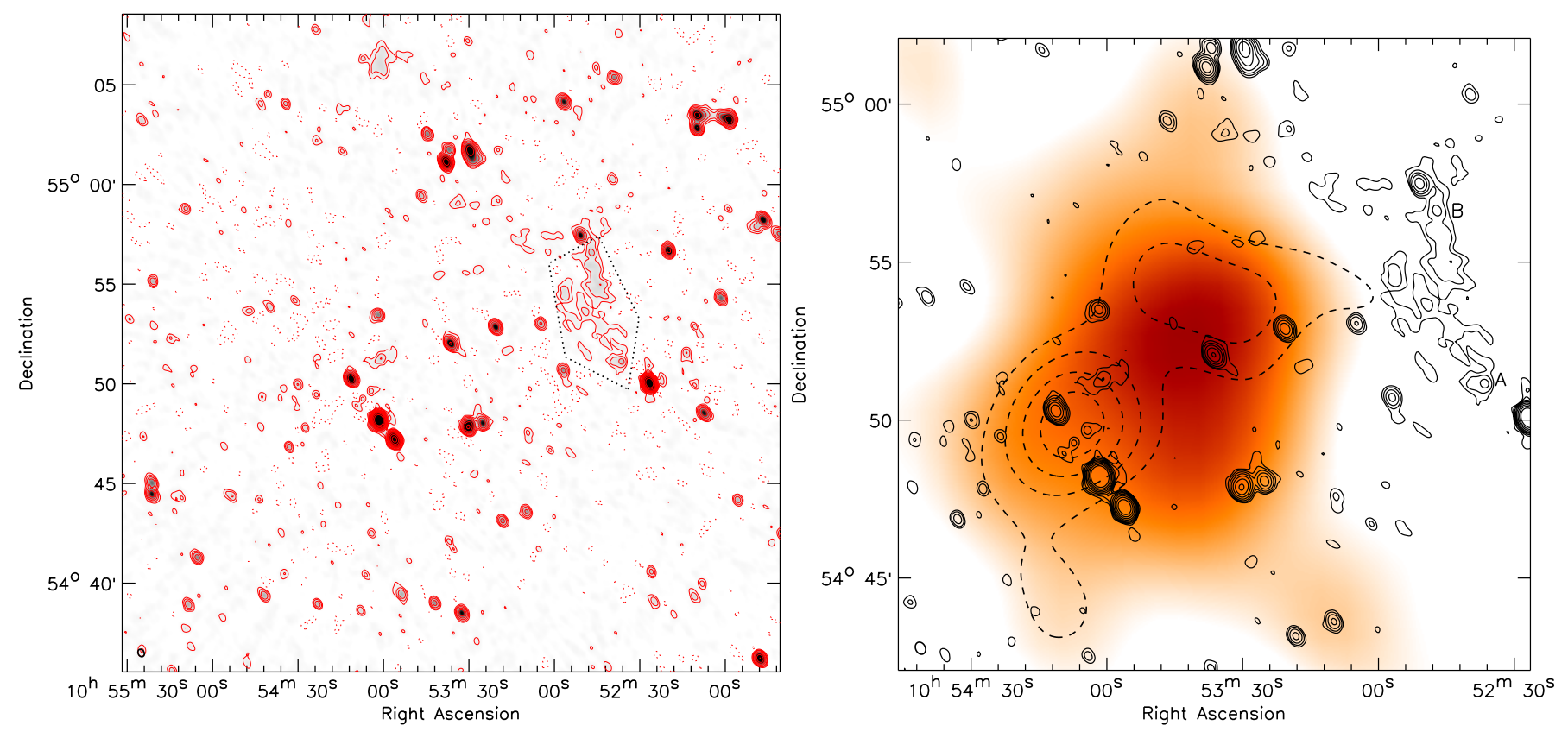

Fig. 21. Left: RXC J1053.7+5452 WSRT $1382 \mathrm{MHz}$ image. Contour levels are drawn as in Fig. 1. Black dotted lines indicate the integration area for the flux measurement. Right: RXC J1053.7+5452 X-ray emission from ROSAT in orange. The original image from the ROSAT All Sky Survey was convolved with a 225" FWHM Gaussian. Solid contours are from the WSRT $1382 \mathrm{MHz}$ image and drawn at levels of $[1,2,4,8, \ldots] \times 4 \sigma_{\text {rms }}$. Dashed contours show the galaxy iso-density distribution derived from the SDSS survey. Contours are drawn at $[1.0,1.2,1.4, \ldots] \times 0.25$ galaxies $\operatorname{arcmin}^{-2}$ selecting only galaxies with $0.05<z_{\text {phot }}<0.1$. Discrete sources embedded in the diffuse emission are alphabetically labeled, see Table 3 .
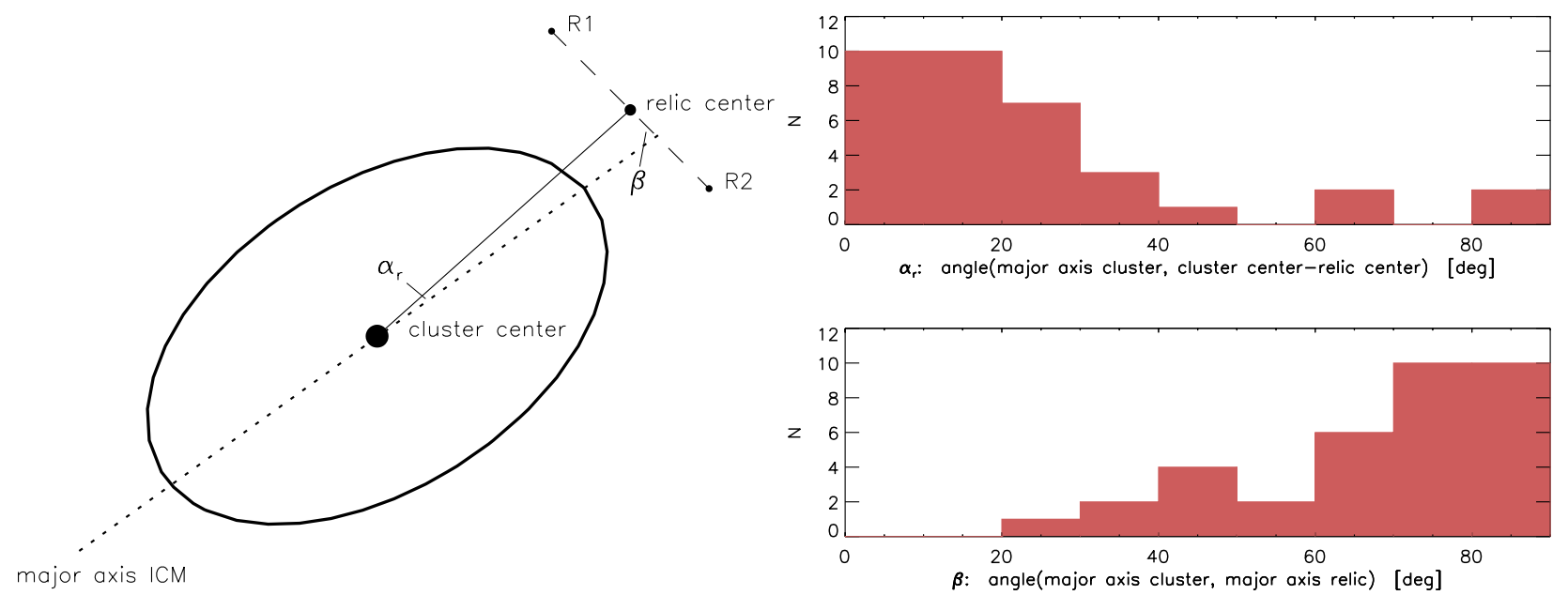

Fig. 22. Left: schematic illustration of the angle between the major axis of the ICM and the line relic center-cluster center $\left(\alpha_{\mathrm{r}}\right)$, and the angle between the relic orientation and major axis of the ICM $(\beta)$, see Table 4. Top right: histogram of angles between the major axis of the X-ray emission and the line connecting the cluster center with the center of the relic. Bottom right: histogram of angles between the major axis of the $\mathrm{X}$-ray emission and the relic's major axis.

limit the NORAS is more than $50 \%$ incomplete, see Fig. 24. The total number of clusters above this flux limit is 540, and the number of clusters with relics is 16 (using the same flux cutoff). From this we find that the currently observed fraction of clusters hosting relics is $3 \%$ in this sample. The list of relics is given in Table 5 and the radio power are plotted as function of redshift are plotted in Fig. 25.

The resulting histograms are displayed in Fig. 26. Although the number of known relics is rather small, and the relics were selected using various methods, there are few interesting trends visible. Apparently, the fraction of clusters with relics increases with the $\mathrm{X}$-ray luminosity, from about a percent at $L_{\mathrm{X}, 0.1-2.4 \mathrm{keV}}=1 \times 10^{44} \mathrm{erg} \mathrm{s}^{-1}$ to more than $10 \%$ above $\sim 1 \times 10^{45} \mathrm{erg} \mathrm{s}^{-1}$. Also, the redshift distribution for cluster with relics is somewhat broader than the corresponding NORASREFLEX sample. Therefore, the chance of finding a relic above a certain flux density increases with redshift for clusters selected from flux-limited X-ray surveys. Since the number of clusters with relics is small this might be a statistical fluctuation. However, given that the average fraction of clusters with relics is $3 \%$, the probability to find four (or even more) in the 26 clusters with $L_{\mathrm{X}}>10^{45} \mathrm{erg} \mathrm{s}^{-1}$ is $0.7 \%$. Hence, a pure statistical 
Table 4. Clusters with radio relics and measured orientations.

\begin{tabular}{lccc}
\hline \hline Cluster name & $z$ & $\begin{array}{c}\alpha_{\mathrm{r}} \\
\text { "location PA" (deg) }\end{array}$ & $\begin{array}{c}\beta \\
\text { "orientation PA" (deg) }\end{array}$ \\
\hline Abell 115 & 0.197 & 45 & 78 \\
Abell 521 & 0.247 & 6 & 64 \\
Abell 523 & 0.100 & 23 & 39 \\
Abell 548b & 0.042 & $87(\mathrm{~A}), 40(\mathrm{~B})$ & $84(\mathrm{~A}), 48(\mathrm{~B})$ \\
Abell 746 & 0.232 & 25 & 56 \\
Abell 1240 & 0.159 & $13(\mathrm{~N}), 15(\mathrm{~S})$ & $85(\mathrm{~N}), 85(\mathrm{~S})$ \\
Abell 1612 & 0.179 & 62 & 69 \\
Abell 1664 & 0.128 & 3 & 78 \\
Abell 2061 & 0.078 & 15 & 77 \\
Abell 2163 & 0.203 & 1 & 67 \\
Abell 2255 & 0.081 & 61 & 38 \\
Abell 2256 & 0.059 & 26 & 77 \\
Abell 2345 & 0.176 & $13(\mathrm{~W}), 24(\mathrm{E})$ & $87(\mathrm{~W}), 43(\mathrm{E})$ \\
Abell 2744 & 0.308 & 20 & 81 \\
Abell 3365 & 0.093 & $20(\mathrm{~W}), 141(\mathrm{E})$ & $82(\mathrm{~W}), 43(\mathrm{E})$ \\
Abell 3376 & 0.046 & $3(\mathrm{~W}), 8(\mathrm{E})$ & $85(\mathrm{~W}), 67(\mathrm{E})$ \\
Abell 3667 & 0.055 & $13(\mathrm{~W}), 0(\mathrm{E})$ & $75(\mathrm{~W}), 75(\mathrm{E})$ \\
Coma cluster & 0.023 & 22 & 60 \\
CIZA J2252.8+5301 & 0.192 & $27(\mathrm{~N}), 4(\mathrm{~S})$ & $67(\mathrm{~N}), 84(\mathrm{~S})$ \\
MACS J0717.5+3745 & 0.555 & 2 & 79 \\
RXC J1053.7+5452 & 0.070 & 19 & 76 \\
RXCJ1314.4-2515 & 0.244 & $30(\mathrm{~W}), 99(\mathrm{E})$ & $26(\mathrm{~W}), 46(\mathrm{E})$ \\
ZwCl 0008.8+5215 & 0.104 & $10(\mathrm{~W}), 11(\mathrm{E})$ & $76(\mathrm{~W}), 85(\mathrm{E})$ \\
ZwCl 2341.1+0000 & 0.270 & $14(\mathrm{~N}), 8(\mathrm{~S})$ & $88(\mathrm{~N}), 63(\mathrm{~S})$ \\
\hline
\end{tabular}

Notes. For double relics two values are measured $\left(\mathrm{N}=\right.$ north, $\mathrm{S}=$ south, $\mathrm{E}=$ east, $\mathrm{W}=$ west). ${ }^{a}$ Identification uncertain (either relic or halo); ${ }^{b}$ A548b: A and B refer to the names used in Feretti et al. (2006).

References. Abell 2255 (main relic only) Pizzo \& de Bruyn (2009); Govoni et al. (2005), MACS J0717.5+3745 van Weeren et al. (2009c); Bonafede et al. (2009a), Abell 548b (2 relics) Feretti et al. (2006), Abell 2256 Clarke \& Ensslin (2006), Abell 521 Giacintucci et al. (2008), Coma Cluster Giovannini et al. (1991); Brown \& Rudnick (2011), Abell 2163 Feretti et al. (2001), Abell 2744 Govoni et al. (2001), Abell 115 and Abell 1664 Govoni et al. (2001). Symmetric double relics included from the literature are: Abell 1240 and Abell 2345 Bonafede et al. (2009b), Abell 3376 Bagchi et al. (2006), Abell 3667 Röttgering et al. (1997), ZwCl 0008.8+5215 van Weeren et al. (2011a), CIZA J2242.8+5301 van Weeren et al. (2010), ZwCl 2341.1+0000 van Weeren et al. (2009a); Giovannini et al. (2010), RXC J1314.4-2515 Venturi et al. (2007).

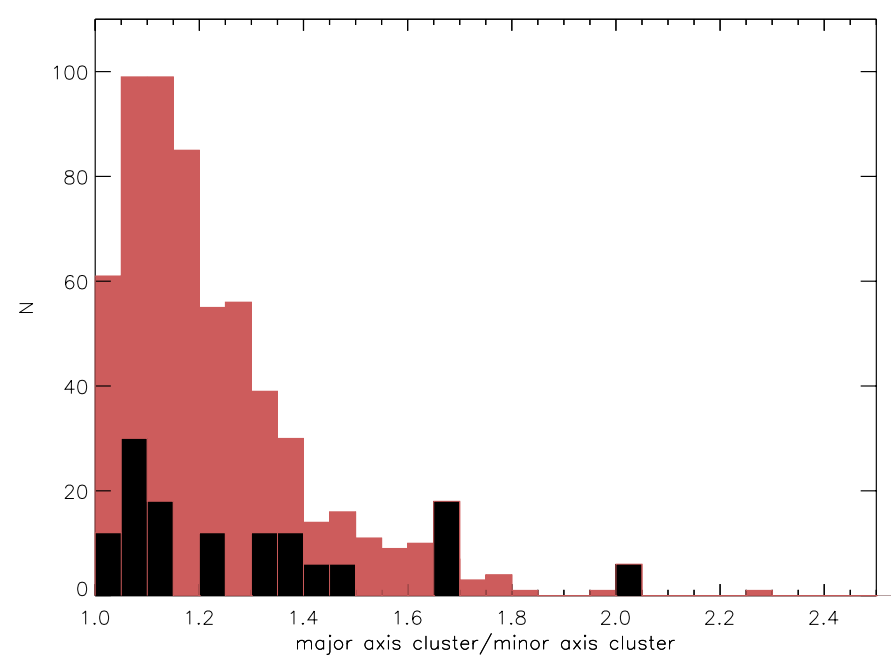

Fig. 23. Histograms showing the distribution of the major and minor axis ratio. The red histogram is for clusters from the NORAS and REFLEX surveys, the black histogram is for clusters containing giant radio relics (see the caption of Fig. 22). The black histogram was scaled by a factor of six for easier comparison with the NORAS-REFLEX sample. Clusters for which the 2 dimensional Gaussian fit did not converge were not included.

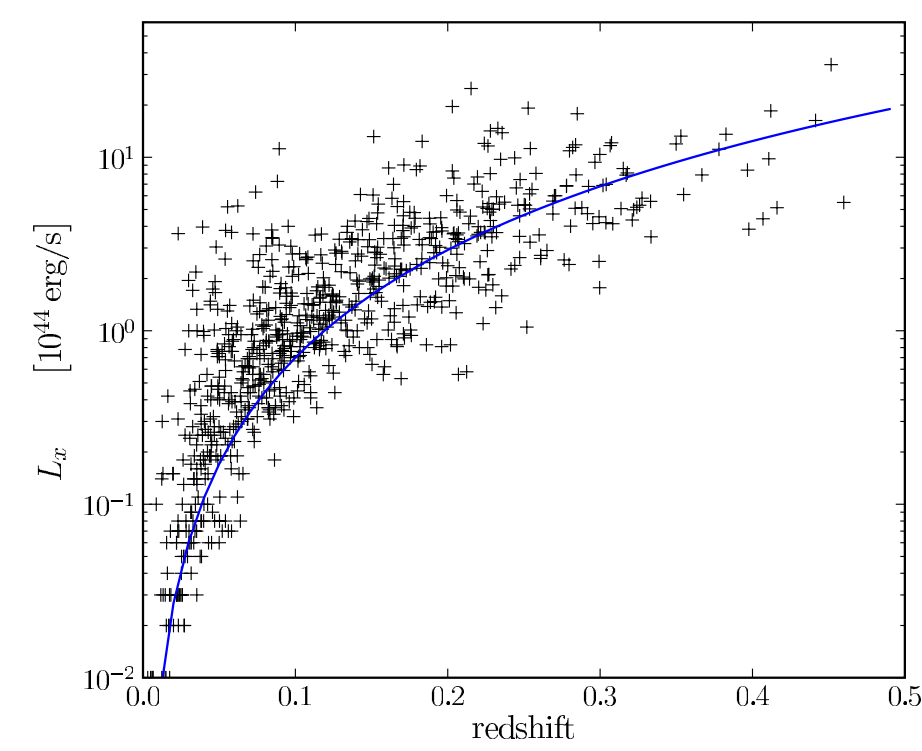

Fig. 24. $L_{X}$-redshift distribution for the NORAS and REFLEX surveys. The solid blue line is the flux cutoff of $3.0 \times 10^{-12} \mathrm{erg} \mathrm{s}^{-1} \mathrm{~cm}^{-2}$ we use for comparison with the relic cluster sample. 
Table 5. Clusters with radio relics in the NORAS and REFLEX surveys

\begin{tabular}{lccc}
\hline \hline Cluster name & $z$ & $\begin{array}{c}P_{1.4 \mathrm{GHz}}(\text { total for cluster) } \\
10^{24} \mathrm{~W} \mathrm{~Hz}^{-1}\end{array}$ & $\begin{array}{c}L_{\mathrm{X}, 0.1-2.4 \mathrm{keV}} \\
10^{44} \mathrm{erg} \mathrm{s}^{-1}\end{array}$ \\
\hline $\mathrm{A} 3376$ & 0.0468 & 1.6 & 1.01 \\
$\mathrm{~A} 2744$ & 0.3066 & 7.7 & 11.68 \\
$\mathrm{~A} 4038^{c}$ & 0.0292 & 0.1 & 1.00 \\
$\mathrm{~A} 0548 \mathrm{~W}^{b}$ & 0.0424 & 0.5 & 0.10 \\
$\mathrm{RXC} \mathrm{J}^{b} 14.4-2515$ & 0.2439 & 8.2 & 9.92 \\
$\mathrm{~A} 133^{c}$ & 0.0569 & 1.1 & 1.40 \\
$\mathrm{~A} 1300$ & 0.3075 & 6.3 & 12.12 \\
$\mathrm{~A} 13^{c}$ & 0.0940 & 0.9 & 1.24 \\
$\mathrm{~A} 2345$ & 0.1760 & 6.2 & 3.91 \\
$\mathrm{~A} 521$ & 0.2475 & 3.4 & 7.44 \\
$\mathrm{~A} 754$ & 0.0542 & 0.04 & 3.79 \\
$\mathrm{~A} 85^{c}$ & 0.0555 & 0.3 & 5.18 \\
$\mathrm{~A} 2163$ & 0.2030 & 2.8 & 19.62 \\
$\mathrm{~A} 1612$ & 0.1797 & 7.9 & 2.41 \\
$\mathrm{~A} 523^{a}$ & 0.1034 & 1.8 & 0.89 \\
Coma cluster & 0.0231 & 0.29 & 3.63 \\
$\mathrm{~A} 781^{b}$ & 0.2952 & 5.8 & 4.15 \\
$\mathrm{~A} 2034^{a}$ & 0.1130 & 1.17 & 3.56 \\
$\mathrm{~A} 1758$ & 0.2799 & 4.1 & 10.90 \\
$\mathrm{~A} 746$ & 0.232 & 6.8 & 3.68 \\
RXC J1053.7+5452 $^{b}$ & 0.0704 & 0.2 & 0.44 \\
A2255 & 0.0809 & 0.8 & 3.08 \\
A2256 & 0.0581 & 4.2 & 3.69 \\
\hline
\end{tabular}

Notes. ${ }^{a}$ Identification uncertain (either relic or halo); ${ }^{b}$ below X-ray flux limit of $F_{\mathrm{X}, 0.1-2.4 \mathrm{keV}}=3 \times 10^{-12} \mathrm{erg} \mathrm{s}^{-1} \mathrm{~cm}^{-2} ;{ }^{c}$ radio phoenix, not included in sample.

References. Abell 754 Macario et al. (2011); Abell 1758 Giovannini et al. (2009); Abell 781 Venturi et al. (2011); Abell 1300 Reid et al. (1999); Abell 13, 85, 133, and 4038 Slee et al. (2001); for other clusters see Table 4.

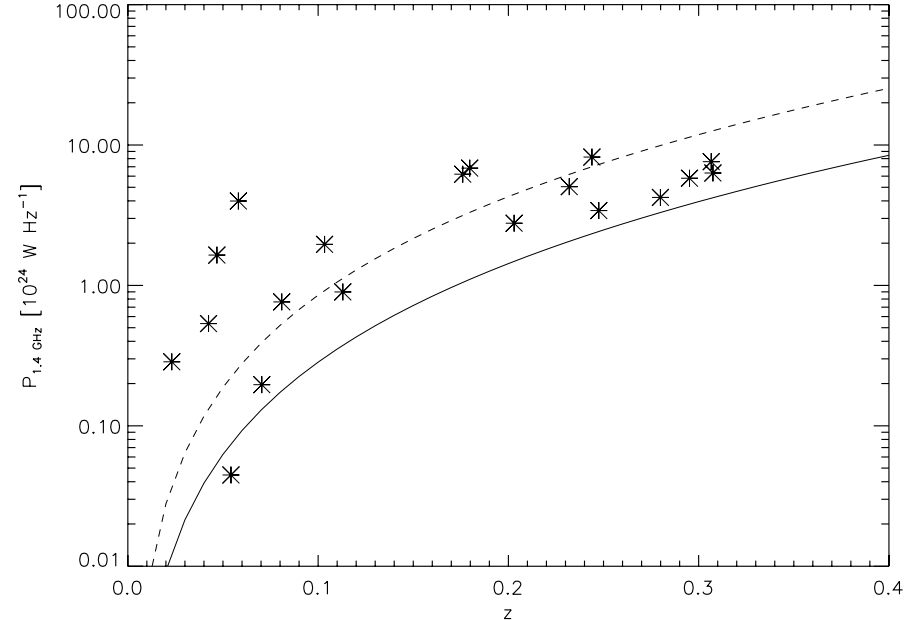

Fig. 25. $P_{1.4 \mathrm{GHz}}$-redshift distribution for the relics in Table 5. The solid line is for a relic flux limit of $10 \mathrm{mJy}$, the dashed line for $30 \mathrm{mJy}$. Radio phoenices are not included.

fluctuation is rather unlikely. This rises the question if selection effects may causes this trend.

As discussed above the NORAS-REFLEX sample is more or less complete up to $3 \times 10^{-12} \mathrm{erg} \mathrm{s}^{-1} \mathrm{~cm}^{-2}$. The relic sample in contrast is probably not complete up to a specific flux limit. For example, large low-surface brightness relics could be missed, as are relics in more distant clusters because they are barely resolved in the NVSS and WENSS surveys images and therefore not easily recognizable. The construction of a flux-limited relic sample is therefore challenging as selection effects due to angular size, morphology and surface brightness need to be properly taken into account. In addition, one needs to properly identify other diffuse radio sources (such as radio halos and giant radio galaxies) to prevent them from ending up in the relic sample. Rudnick \& Lemmerman (2009) give a good overview of some of the problems involved in constructing these samples. The important question is whether there is any systematic effect which leads to a preferential detection of relics in luminous clusters. Since the luminous clusters are on average at higher redshift, see Fig. 24, relic detection is less affected by a too low surface brightness. However, it is possibly affected by resolution effects. This will only decrease the detection probability at higher redshift (luminosity) and hence there is no evident reason why relics in more luminous clusters should have a better chance of being discovered.

Skillman et al. (2011) found in simulations, based on DSA, that the radio power of clusters with relics scatters largely for a given $\mathrm{X}$-ray luminosity. In addition, they found that the mean radio power strongly correlates with the X-ray luminosity. Hence, the fraction of clusters with relics should increase with X-ray luminosity. To compute the actual fractions many factors need to be considered, namely the X-ray flux limit, the discovery probability for radio relics, the radio power distribution of relics and the abundance of clusters as function of X-ray luminosity and redshift. In Nuza et al. (2011) we cary out this analysis in detail. We postulate a radio relic probability density based on numerical simulations $p\left(P, M_{\mathrm{vir}}, z, v_{\mathrm{obs}}\right)$, where $P$ is the radio luminosity, $M_{\mathrm{vir}}$ is the virial mass of the cluster and $v_{\mathrm{obs}}$ is the observing frequency. We convolve this with the cosmological abundance of dark matter halos. As a result we indeed find that the fraction of radio relics in the NORAS-REFLEX sample should increase 

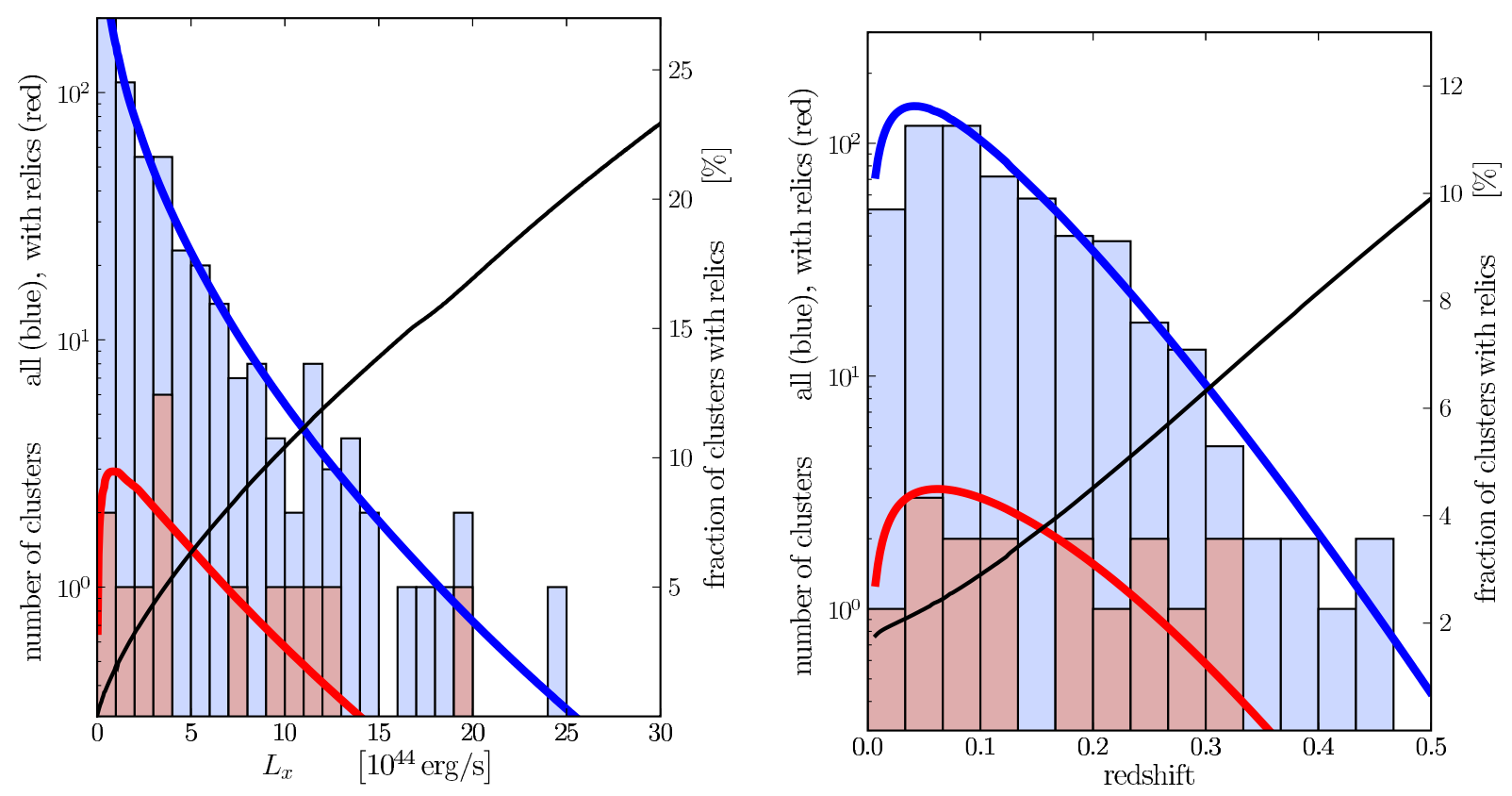

Fig. 26. Histograms showing the X-ray luminosity (left) and redshift (right) distribution. Blue histograms shows the NORAS-REFLEX sample, red the relic cluster sample. The solid blue line displays the predicted luminosity/redshift distributions from Nuza et al. (2011) for clusters with fluxes $>3.0 \times 10^{-12} \mathrm{erg} \mathrm{s}^{-1} \mathrm{~cm}^{-2}$, while the solid red line is the prediction for clusters hosting relics in the simulation. The fraction of clusters with relics is given by the black solid line (the ratio of the blue and red lines).

with both the X-ray luminosity and the redshift, see Fig. 26 (solid lines).

The reason why in simulations more massive clusters show on average much brighter radio relics is unclear. We speculate that multiple aspects contribute: temperature and density are higher and shock fronts are larger in more massive clusters. It seems also likely that mergers of more massive clusters result in higher Mach numbers. Since according to the sub-grid models used in the simulation the radio emission strongly increases with Mach number (Hoeft \& Brüggen 2007), a rather small increase of the Mach numbers would have a large effect on the resulting radio luminosity. (We note that the simulation of Hoeft \& Brüggen 2007, is also based on DSA.) Finally, the merger rate increases with redshift. However, it needs to be clarified from simulations why an increase of the radio power with $\mathrm{X}$-ray luminosity is expected. The fractions of relics in X-ray selected cluster samples is therefore a powerful tool to put constraints on the models used in the simulations, and hence on the evolution of magnetic fields and on particle acceleration in the ICM.

\subsection{X-ray peak and galaxy distribution separation}

Cluster mergers are thought to decouple the baryonic matter component from the dark matter (DM). This causes and offset between the gravitational center (measured from lensing) and $\mathrm{X}$-ray center of the cluster. A clear example is the "Bullet cluster" (e.g., Clowe et al. 2006), but the effect has also been measured for other clusters (e.g., Shan et al. 2010). Radio relics and halos are mostly found in merger cluster and are thus good candidates to measure this effect. We do not have lensing measurements for the clusters in our sample, but simply taking the galaxy distribution as a proxy for the dark matter distribution we note offsets between the X-ray emission from ROSAT and the galaxy distribution in the clusters A2034, A3365, A2061, A523 and RXC J1053.7+5452 for example. These clusters would therefore make interesting targets for future X-ray and lensing observations.

\section{Conclusions}

We have presented WSRT, VLA and GMRT observations of galaxy clusters with diffuse emission selected from the WENSS and NVSS surveys. We find peripheral radio relics in the clusters Abell 1612, Abell 746 and CIZA J0649.3+1801 and a smaller relic Abell 2034. Abell 3365 seems to host a double radio relic system. Our observations reveal radio halos in the clusters Abell 746 and RX J0107.8+5408. We confirm the presence of radio relics in Abell 2061, RXC J1053.7+5452 and diffuse emission in Abell 523 and Abell 2034 (for which the classification is uncertain). In addition, we detect the radio halo Abell 697 providing additional flux measurements around $1.4 \mathrm{GHz}$.

By constructing a sample of 35 relics we have found that relics are generally located along the major axis (which can be used as a proxy for the merger axis) of the cluster's elongated ICM. Their orientation is mostly perpendicular to this major axis. The distribution of the major-minor axis ratios for the relic cluster sample is broader than that of the NORAS-REFLEX sample. These results are consistent with the scenario that relics trace shock waves which form along the merger axis of clusters. We also compared the redshift and X-ray luminosity distributions of clusters with relics to a sample of clusters from the NORAS and REFLEX surveys. Interestingly, we find indications that the observed fraction of clusters hosting relics increases with X-ray luminosity and redshift. However, selection biases for radio relics play a role and this needs to be investigated further. The significantly improved sensitivity of upcoming radio telescopes (e.g., the EVLA and LOFAR) will allow to find many more radio relics. Correlating this larger relic sample with cluster samples selected at X-rays wavelengths will provide a powerful tool to constrain the evolution of relics in galaxy clusters. 
Acknowledgements. We would like to thank the anonymous referee for useful comments. We thank the staff of the GMRT who have made these observations possible. The GMRT is run by the National Centre for Radio Astrophysics of the Tata Institute of Fundamental Research. The Westerbork Synthesis Radio Telescope is operated by ASTRON (Netherlands Institute for Radio Astronomy) with support from the Netherlands Foundation for Scientific Research (NWO). The National Radio Astronomy Observatory is a facility of the National Science Foundation operated under cooperative agreement by Associated Universities, Inc. The William Herschel Telescope and Isaac Newton Telescope are operated on the island of La Palma by the Isaac Newton Group in the Spanish Observatorio del Roque de los Muchachos of the Instituto de Astrofisica de Canarias. The National Radio Astronomy Observatory is a facility of the National Science Foundation operated under cooperative agreement by Associated Universities, Inc. R.J.v.W. acknowledges funding from the Royal Netherlands Academy of Arts and Sciences. M.B. and M.H. acknowledge support by the research group FOR 1254 funded by the Deutsche Forschungsgemeinschaft.

\section{References}

Abazajian, K. N., Adelman-McCarthy, J. K., Agüeros, M. A., et al. 2009, ApJS, 182,543

Abell, G. O., Corwin, Jr., H. G., \& Olowin, R. P. 1989, ApJS, 70, 1

Aguerri, J. A. L., Sánchez-Janssen, R., \& Muñoz-Tuñón, C. 2007, A\&A, 471, 17

Athreya, R. 2009, ApJ, 696, 885

Axford, W. I., Leer, E., \& Skadron, G. 1977, in International Cosmic Ray Conference, 11,132

Baars, J. W. M., Genzel, R., Pauliny-Toth, I. I. K., \& Witzel, A. 1977, A\&A, 61, 99

Bacchi, M., Feretti, L., Giovannini, G., \& Govoni, F. 2003, A\&A, 400, 465 Bagchi, J., Durret, F., Neto, G. B. L., \& Paul, S. 2006, Science, 314, 791

Barrena, R., Boschin, W., Girardi, M., \& Spolaor, M. 2007, A\&A, 467, 37 Becker, R. H., White, R. L., \& Helfand, D. J. 1995, ApJ, 450, 559

Bell, A. R. 1978a, MNRAS, 182, 147

Bell, A. R. 1978b, MNRAS, 182, 443

Bertin, E., \& Arnouts, S. 1996, A\&AS, 117, 393

Blandford, R., \& Eichler, D. 1987, Phys. Rep., 154, 1

Blandford, R. D., \& Ostriker, J. P. 1978, ApJ, 221, L29

Blasi, P., \& Colafrancesco, S. 1999, Astropart. Phys., 12, 169

Böhringer, H., Voges, W., Huchra, J. P., et al. 2000, ApJS, 129, 435

Böhringer, H., Schuecker, P., Guzzo, L., et al. 2004, A\&A, 425, 367

Bonafede, A., Feretti, L., Giovannini, G., et al. 2009a, A\&A, 503, 707

Bonafede, A., Giovannini, G., Feretti, L., Govoni, F., \& Murgia, M. 2009b, A\&A, 494, 429

Bonafede, A., Govoni, F., Feretti, L., et al. 2011, A\&A, 530, A24

Briggs, D. S. 1995, Ph.D. Thesis, New Mexico Institute of Mining Technology, Socorro, New Mexico, USA

Brunetti, G., Setti, G., Feretti, L., \& Giovannini, G. 2001, MNRAS, 320, 365

Brunetti, G., Giacintucci, S., Cassano, R., et al. 2008, Nature, 455, 944

Brunetti, G., Cassano, R., Dolag, K., \& Setti, G. 2009, A\&A, 507, 661

Brown, S., \& Rudnick, L. 2011, MNRAS, 412, 2

Brown, S., Duesterhoeft, J., \& Rudnick, L. 2011, ApJ, 727, L25

Buote, D. A. 2001, ApJ, 553, L15

Cassano, R., \& Brunetti, G. 2005, MNRAS, 357, 1313

Cassano, R., Brunetti, G., \& Setti, G. 2006, MNRAS, 369, 1577

Cassano, R., Brunetti, G., Setti, G., Govoni, F., \& Dolag, K. 2007, MNRAS, 378, 1565

Cassano, R., Brunetti, G., Venturi, T., et al. 2008, A\&A, 480, 687

Cassano, R., Brunetti, G., Röttgering, H. J. A., \& Brüggen, M. 2010a, A\&A, 509, A68

Cassano, R., Ettori, S., Giacintucci, S., et al. 2010b, ApJ, 721, L82

Cavagnolo, K. W., Donahue, M., Voit, G. M., \& Sun, M. 2009, ApJS, 182, 12

Clarke, T. E., \& Ensslin, T. A. 2006, AJ, 131, 2900

Clowe, D., Bradač, M., Gonzalez, A. H., et al. 2006, ApJ, 648, L109

Condon, J. J., Cotton, W. D., Greisen, E. W., et al. 1998, AJ, 115, 1693

Cornwell, T. J., \& Perley, R. A. 1992, A\&A, 261, 353

Cotton, W. D. 2008, PASP, 120, 439

Crawford, C. S., Edge, A. C., Fabian, A. C., et al. 1995, MNRAS, 274, 75

Dennison, B. 1980, ApJ, 239, L93

Dolag, K., \& Enßlin, T. A. 2000, A\&A, 362, 151

Donnert, J., Dolag, K., Brunetti, G., Cassano, R., \& Bonafede, A. 2010a, MNRAS, 401, 47

Donnert, J., Dolag, K., Cassano, R., \& Brunetti, G. 2010b, MNRAS, 407, 1565
Drury, L. O. 1983, Reports on Progress in Physics, 46, 973

Ebeling, H., Edge, A. C., Bohringer, H., et al. 1998, MNRAS, 301, 881

Ebeling, H., Mullis, C. R., \& Tully, R. B. 2002, ApJ, 580, 774

Enßlin, T. A., \& Brüggen, M. 2002, MNRAS, 331, 1011

Enßlin, T. A., \& Gopal-Krishna. 2001, A\&A, 366, 26

Ensslin, T. A., Biermann, P. L., Klein, U., \& Kohle, S. 1998, A\&A, 332, 395

Feretti, L., Fusco-Femiano, R., Giovannini, G., \& Govoni, F. 2001, A\&A, 373, 106

Feretti, L., Schuecker, P., Böhringer, H., Govoni, F., \& Giovannini, G. 2005, A\&A, 444, 157

Feretti, L., Bacchi, M., Slee, O. B., et al. 2006, MNRAS, 368, 544

Giacintucci, S., Venturi, T., Macario, G., et al. 2008, A\&A, 486, 347

Giovannini, G., \& Feretti, L. 2000, New Astronomy, 5, 335

Giovannini, G., Feretti, L., \& Stanghellini, C. 1991, A\&A, 252, 528

Giovannini, G., Tordi, M., \& Feretti, L. 1999, New Astron., 4, 141

Giovannini, G., Bonafede, A., Feretti, L., et al. 2009, A\&A, 507, 1257

Giovannini, G., Bonafede, A., Feretti, L., Govoni, F., \& Murgia, M. 2010, A\&A, 511, L5

Giovannini, G., Feretti, L., Girardi, M., et al. 2011, A\&A, 530, L5

Girardi, M., Boschin, W., \& Barrena, R. 2006, A\&A, 455, 45

Govoni, F., \& Feretti, L. 2004, Int. J. Mod. Phys. D, 13, 1549

Govoni, F., Feretti, L., Giovannini, G., et al. 2001, A\&A, 376, 803

Govoni, F., Markevitch, M., Vikhlinin, A., et al. 2004, ApJ, 605, 695

Govoni, F., Murgia, M., Feretti, L., et al. 2005, A\&A, 430, L5

Hales, S. E. G., Riley, J. M., Waldram, E. M., Warner, P. J., \& Baldwin, J. E. 2007, MNRAS, 382, 1639

Hoeft, M., \& Brüggen, M. 2007, MNRAS, 375, 77

Jaffe, W. J. 1977, ApJ, 212, 1

Jeltema, T. E., \& Profumo, S. 2011, ApJ, 728, 53

Jones, F. C., \& Ellison, D. C. 1991, Space Sci. Rev., 58, 259

Kang, H., \& Ryu, D. 2011, ApJ, 734, 18

Katgert, P., Mazure, A., Perea, J., et al. 1996, A\&A, 310, 8

Kempner, J. C., \& Sarazin, C. L. 2001, ApJ, 548, 639

Kempner, J. C., Sarazin, C. L., \& Markevitch, M. 2003, ApJ, 593, 291

Keshet, U. 2010, ArXiv e-prints

Keshet, U., \& Loeb, A. 2010, ApJ, 722, 737

Kocevski, D. D., Ebeling, H., Mullis, C. R., \& Tully, R. B. 2007, ApJ, 662, 224

Koester, B. P., McKay, T. A., Annis, J., et al. 2007, ApJ, 660, 239

Krymskii, G. F. 1977, Akademiia Nauk SSSR Doklady, 234, 1306

Liang, H., Hunstead, R. W., Birkinshaw, M., \& Andreani, P. 2000, ApJ, 544, 686

Macario, G., Venturi, T., Brunetti, G., et al. 2010, A\&A, 517, A43

Macario, G., Markevitch, M., Giacintucci, S., et al. 2011, ApJ, 728, 82

Malkov, M. A., \& O'C Drury, L. 2001, Reports on Progress in Physics, 64, 429

Marini, F., Bardelli, S., Zucca, E., et al. 2004, MNRAS, 353, 1219

Markevitch, M., Govoni, F., Brunetti, G., \& Jerius, D. 2005, ApJ, 627, 733

Maughan, B. J., Jones, C., Forman, W., \& Van Speybroeck, L. 2008, ApJS, 174, 117

Mazure, A., Katgert, P., den Hartog, R., et al. 1996, A\&A, 310, 31

Miniati, F., Ryu, D., Kang, H., et al. 2000, ApJ, 542, 608

Noordam, J. E. 2004, in SPIE Conf. Ser. 5489, ed. J. M. Oschmann, Jr., 817

Nuza, S. E., Hoeft, M., van Weeren, R. J., Gottlöber, S., \& Yepes, G. 2011, MNRAS, submitted

Oegerle, W. R., \& Hill, J. M. 2001, AJ, 122, 2858

Paul, S., Iapichino, L., Miniati, F., Bagchi, J., \& Mannheim, K. 2011, ApJ, 726, 17

Perley, R. A. 1989, in Synthesis Imaging in Radio Astronomy, ed. R. A. Perley, F. R. Schwab, \& A. H. Bridle, ASP Conf. Ser., 6, 259

Perley, R. T., \& Taylor, G. B. 1999, VLA Calibrator Manual, Tech. Rep., NRAO

Petrosian, V. 2001, ApJ, 557, 560

Pizzo, R. F., \& de Bruyn, A. G. 2009, A\&A, 507, 639

Popesso, P., Böhringer, H., Brinkmann, J., Voges, W., \& York, D. G. 2004, A\&A, 423, 449

Reid, A. D., Hunstead, R. W., Lemonon, L., \& Pierre, M. M. 1999, MNRAS, 302,571

Rengelink, R. B., Tang, Y., de Bruyn, A. G., et al. 1997, A\&AS, 124, 259

Ricker, P. M., \& Sarazin, C. L. 2001, ApJ, 561, 621

Rines, K., \& Diaferio, A. 2006, AJ, 132, 1275

Roettiger, K., Burns, J. O., \& Stone, J. M. 1999, ApJ, 518, 603

Röttgering, H. J. A., Wieringa, M. H., Hunstead, R. W., \& Ekers, R. D. 1997, MNRAS, 290, 577

Roy, J., Gupta, Y., Pen, U., et al. 2010, Exp. Astron., 28, 25

Rudnick, L., \& Lemmerman, J. A. 2009, ApJ, 697, 1341

Shan, H., Qin, B., Fort, B., et al. 2010, MNRAS, 406, 1134

Skillman, S. W., Hallman, E. J., O'Shea, B. W., et al. 2011, ApJ, 735, 96 
A\&A 533, A35 (2011)

Slee, O. B., Roy, A. L., Murgia, M., Andernach, H., \& Ehle, M. 2001, AJ, 122, 1172

Small, T. A., Ma, C., Sargent, W. L. W., \& Hamilton, D. 1998, ApJ, 492, 45 Struble, M. F., \& Rood, H. J. 1999, ApJS, 125, 35

van Weeren, R. J., Röttgering, H. J. A., Bagchi, J., et al. 2009a, A\&A, 506, 1083 van Weeren, R. J., Röttgering, H. J. A., Brüggen, M., \& Cohen, A. 2009b, A\&A, 508,75

van Weeren, R. J., Röttgering, H. J. A., Brüggen, M., \& Cohen, A. 2009c, A\&A, 505,991

van Weeren, R. J., Röttgering, H. J. A., Brüggen, M., \& Hoeft, M. 2010, Science, 330,347 van Weeren, R. J., Hoeft, M., Röttgering, H. J. A., et al. 2011a, A\&A, 528, A38

van Weeren, R. J., Röttgering, H. J. A., \& Brüggen, M. 2011b, A\&A, 527, A114

Venturi, T., Giacintucci, S., Brunetti, G., et al. 2007, A\&A, 463, 937

Venturi, T., Giacintucci, S., Dallacasa, D., et al. 2008, A\&A, 484, 327

Venturi, T., Giacintucci, G., Dallacasa, D., et al. 2011, MNRAS, L251

Voges, W., Aschenbach, B., Boller, T., et al. 1999, A\&A, 349, 389

Voges, W., Aschenbach, B., Boller, T., et al. 2000, IAU Circ., 7432, 1

Wardle, J. F. C., \& Kronberg, P. P. 1974, ApJ, 194, 249

White, D. A. 2000, MNRAS, 312, 663 